Article

\title{
Solution of Inhomogeneous Differential Equations with Polynomial Coefficients in Terms of the Green's Function
}

\author{
Tohru Morita ${ }^{1, *}$ and Ken-ichi Sato ${ }^{2}$ \\ 1 Graduate School of Information Sciences, Tohoku University, Sendai 980-8577, Japan \\ 2 College of Engineering, Nihon University, Koriyama 963-8642, Japan; kensatokurume@ybb.ne.jp \\ * Correspondence: senmm@jcom.home.ne.jp; Tel.: +81-22-278-6186
}

Received: 30 September 2017; Accepted: 6 November 2017; Published: 10 November 2017

\begin{abstract}
The particular solutions of inhomogeneous differential equations with polynomial coefficients in terms of the Green's function are obtained in the framework of distribution theory. In particular, discussions are given on Kummer's and the hypergeometric differential equation. Related discussions are given on the particular solution of differential equations with constant coefficients, by the Laplace transform.
\end{abstract}

Keywords: Green's function; distribution theory; particular solution; Kummer's differential equation; hypergeometric differential equation; Laplace transform

\section{Introduction}

In our recent papers [1,2], we are concerned with the solution of Kummer's and hypergeometric differential equations, where complementary solutions expressed by the confluent hypergeometric series and the hypergeometric series, respectively, are obtained, by using the Laplace transform, its analytic continuation, distribution theory and the fractional calculus.

It is the purpose of the present paper, to give the formulas which give the particular solutions of those equations with inhomogeneous term in terms of the Green's function. The differential equation satisfied by the Green's function is expressed with the aid of Dirac's delta function, which is defined in distribution theory, and hence the presentation in distribution theory is adopted.

Let

$$
p_{K}(t, s):=t \cdot s^{2}+(c-b t) s-a b,
$$

where $a \in \mathbb{C}, b \in \mathbb{C}$ and $c \in \mathbb{C}$. Then Kummer's differential equation with an inhomogeneous term is given by

$$
p_{K}\left(t, \frac{d}{d t}\right) u(t):=t \cdot \frac{d^{2}}{d t^{2}} u(t)+(c-b t) \cdot \frac{d}{d t} u(t)-a b \cdot u(t)=f(t), \quad t>0 .
$$

If $c \notin \mathbb{Z}$, the basic complementary solutions of Equation (2) are given by

$$
\begin{aligned}
& K_{1}(t):={ }_{1} F_{1}(a ; c ; b t), \\
& K_{2}(t):=t^{1-c} \cdot{ }_{1} F_{1}(a-c+1 ; 2-c ; b t) .
\end{aligned}
$$

Here ${ }_{1} F_{1}(a ; c ; z)=\sum_{k=0}^{\infty} \frac{(a)_{k}}{k !(c)_{k}} z^{k}$ is the confluent hypergeometric series, $(a)_{k}=\prod_{l=0}^{k-1}(a+l)$ for $k \in \mathbb{Z}_{>0}$, and $(a)_{0}=1$. 
We use $\mathbb{R}, \mathbb{C}$ and $\mathbb{Z}$ to denote the sets of all real numbers, of all complex numbers and of all integers, respectively. We also use $\mathbb{R}_{>r}:=\{x \in \mathbb{R} \mid x>r\}, \mathbb{R}_{\geq r}:=\{x \in \mathbb{R} \mid x \geq r\}$ for $r \in \mathbb{R},+\mathbb{C}:=$ $\{z \in \mathbb{C} \mid \operatorname{Re} z>0\}, \mathbb{Z}_{>a}:=\{n \in \mathbb{Z} \mid n>a\}, \mathbb{Z}_{<b}:=\{n \in \mathbb{Z} \mid n<b\}$ and $\mathbb{Z}_{[a, b]}:=\{n \in \mathbb{Z} \mid a \leq n \leq b\}$ for $a \in \mathbb{Z}$ and $b \in \mathbb{Z}$ satisfying $a<b$. We use Heaviside's step function $H(t)$, which is defined by

$$
H(t)= \begin{cases}1, & t>0 \\ 0, & t \leq 0\end{cases}
$$

and when $f(t)$ is defined on $\mathbb{R}_{>\tau}, f(t) H(t-\tau)$ is equal to $f(t)$ for $t>\tau$ and to 0 for $t \leq \tau$.

When $c \in \mathbb{C}$ satisfies $\operatorname{Re}(1-c)>-1$, the solution $K_{2}(t)$ has the Laplace transform:

$$
\hat{K}_{2}(s)=\mathcal{L}\left[K_{2}(t)\right]:=\int_{0}^{\infty} K_{2}(t) e^{-s t} d t,
$$

and is obtained by solving the Laplace transform of Equation (2). In [1,2], we confirm that the solution $K_{2}(t)$ is obtained by using an analytic continuation of the Laplace transform (AC-Laplace transform) for all nonzero values of $c \in \mathbb{C} \backslash \mathbb{Z}_{>0}$.

The complementary solution of the hypergeometric differential equation, corresponding to $K_{2}(t)$, is found to be obtained by using the Laplace transform series, where the Laplace transforms of the solutions are expresssed by a series of powers of $s^{-1}$ multipied by a power of $s$, which has zero range of convergence. In fact, the series is the asymptotic expansion of Kummer's function $U(a, b, z)([3]$, Section 13.5), which is discussed also in [4]. Even in that case, by the term-by-term inverse Laplace transform, we obtain the desired result. The calculation was justified by distribution theory [2].

In the present paper, we present the solutions giving particular solutions of Kummer's and the hypergeometric differential equation in terms of the Green's function with the aid of distribution theory.

In Section 2, we present the formulas in distribution theory, which are given in the book of Zemanian ([5], Section 6.3), where the particular solution of differential equation with constant coefficients is obtained. We use them in giving the particular solution of differential equation with polynomial coefficients in terms of the Green's function in Section 3, and the solutions are obtained by this method for Kummer's and the hypergeometric differential equation in Sections 4 and 5 , respectively.

In Section 2.2, a formulation of the Laplace transform based on distribution theory is given, which is related with the one in ([5], Section 8.3). The particular solution of differential equation with constant coefficients is obtained by using the AC-Laplace transform in Section 6, which is compared with the formulation of the solution in distribution theory given in Section 3, where the Green's function plays an important role. In Sections 6.1 and 6.2, the solutions of differentaal equations of the first order and of the second order, respectively, are given.

We mention here that there are papers in which systematic study is made on polynomial solutions of inhomogeneous differential equation with polynomial coefficients; see [6] and its references. In the present paper, we are concerned with infinite series solutions. In our preceding papers $[7,8]$ stimulated by Yosida's works $[9,10]$ on Laplace's differential equations, of which typical one is Kummer's equation, we sudied the solution of Kummer's equation and a simple fractional differential equation on the basis of fractional calculus and distribution theory. In [1], we discussed it in terms of the AC-Laplace transform. In [4], we applied the arguments in [1] to the solution of the homogeneous hypergeometric equation. We now discuss the solution of inhomogeneous equations in terms of the Green's function and distribution theory. In $[11,12]$, the solution of inhomogeneous differential equation with constant coefficients is discussed in terms of the Green's function and distribution theory. In Section 6, we discuss it in terms of the Green's function and the AC-Laplace transform, where we obtain the solution which is not obtained with the aid of the usual Laplace transform. 


\section{Preliminaries on Distribution Theory}

Distributions in the space $\mathcal{D}^{\prime}$ are first introduced in [5,13-15]. The distributions are either regular ones or their derivatives. A regular distribution $\tilde{f}$ in $\mathcal{D}^{\prime}$ corresponds to a function $f$ which belongs to $\mathcal{L}_{\text {loc }}^{1}(\mathbb{R})$. Here $\mathcal{L}_{\text {loc }}^{1}(\mathbb{R})$ denotes the class of functions which are locally integrable on $\mathbb{R}$. A distribution $\tilde{h}$, which is not a regular one, is expressed as $\tilde{h}(t)=D^{n} \tilde{f}(t)$, by $n \in \mathbb{Z}_{>0}$ and a regular distribution $\tilde{f} \in \mathcal{D}^{\prime}$.

The space $\mathcal{D}$, that is dual to $\mathcal{D}^{\prime}$, is the space of testing functions, which are infinitely differentiable on $\mathbb{R}$ and have a compact support.

Definition 1. A distribution $\tilde{h} \in \mathcal{D}^{\prime}$ is a functional, to which $\langle\tilde{h}, \phi\rangle \in \mathbb{C}$ is associated with every $\phi \in \mathcal{D}$. Let $\tilde{f}$ be the regular distribution which corresponds to a function $f \in \mathcal{L}_{\text {loc }}^{1}(\mathbb{R})$. Then (i):

$$
\langle\tilde{f}, \phi\rangle=\int_{-\infty}^{\infty} f(t) \phi(t) d t
$$

and (ii): if $n \in \mathbb{Z}_{>0}, \tilde{h}(t)=D^{n} \tilde{f}(t)$ is such a distribution belonging to $\mathcal{D}^{\prime}$, that

$$
\langle\tilde{h}, \phi\rangle=\left\langle D^{n} \tilde{f}, \phi\right\rangle=\left\langle\tilde{f}, D_{W}^{n} \phi\right\rangle=\int_{-\infty}^{\infty} f(t)\left[D_{W}^{n} \phi(t)\right] d t,
$$

where $D_{W}^{n} \phi(t)=(-1)^{n} \frac{d^{n}}{d t^{n}} \phi(t)$.

Lemma 1. Let $\tilde{f}$ be as in Definition 1 , and $g(t):=\frac{d^{n}}{d t^{n}} f(t)=f^{(n)}(t) \in \mathcal{L}_{l o c}^{1}(\mathbb{R})$, and the distribution which corresponds to $g(t)=f^{(n)}(t)$, be $\tilde{g}(t)$. Then $\tilde{g}(t)=D^{n} \tilde{f}(t)$.

Proof. By using Equations (7) and (8), we have

$$
\langle\tilde{g}, \phi\rangle=\int_{-\infty}^{\infty}\left[\frac{d^{n}}{d t^{n}} f(t)\right] \phi(t) d t=\int_{-\infty}^{\infty} f(t)\left[D_{W}^{n} \phi(t)\right] d t=\left\langle D^{n} \tilde{f}, \phi\right\rangle .
$$

Lemma 2. $D^{n}$ for $n \in \mathbb{Z}_{>-1}$ are operators in the space $\mathcal{D}^{\prime}$.

Definition 2. Let $H(t)$ be defined by Equation (5), $\tau \in \mathbb{R}$, and $\psi(t)$ be such that $\psi(t) H(t-\tau) \in \mathcal{L}_{\text {loc }}^{1}(\mathbb{R})$. Then the regular distributions which correspond to $H(t-\tau)$ and $\psi(t) H(t-\tau)$ are denoted by $\tilde{H}(t-\tau)$ and $\psi(t) \tilde{H}(t-\tau)$, respectively.

Lemma 3. Let $\tau \in \mathbb{R}$, and $\psi(t)$ be such that $\psi(\tau)=0$ and $\frac{d}{d t}[\psi(t) H(t-\tau)]=\left[\frac{d}{d t} \psi(t)\right] H(t-\tau) \in$ $\mathcal{L}_{\text {loc }}^{1}(\mathbb{R})$. Then

$$
D[\psi(t) \tilde{H}(t-\tau)]=\left[\frac{d}{d t} \psi(t)\right] \tilde{H}(t-\tau) .
$$

Proof. We confirm this with the aid of Lemma 1 and Definition 2.

Dirac's delta function $\delta(t)$ is defined by

$$
\delta(t)=D \tilde{H}(t)
$$

Lemma 4. Let $\tau \in \mathbb{R}$, and $\psi(t)$ be such that $\frac{d}{d t} \psi(t) \cdot H(t-\tau) \in \mathcal{L}_{\text {loc }}^{1}(\mathbb{R})$. Then

$$
D[\psi(t) \tilde{H}(t-\tau)]= \begin{cases}\frac{d}{d t} \psi(t) \cdot \tilde{H}(t-\tau), & \psi(\tau)=0 \\ \frac{d}{d t} \psi(t) \cdot \tilde{H}(t-\tau)+\psi(\tau) \delta(t-\tau), & \psi(\tau) \neq 0 .\end{cases}
$$


Proof. The lhs is expressed as

$$
D[\psi(t) \tilde{H}(t-\tau)]=D[[\psi(t)-\psi(\tau)] \tilde{H}(t-\tau)]+\psi(\tau) D \tilde{H}(t-\tau) .
$$

From this, we obtain Equation (12), with the aid of Lemma 3 and Equation (11).

Corollary 1. Let $\tau \in \mathbb{R}$, and $\psi(t)$ be such that $\frac{d^{2}}{d t^{2}} \psi(t) \cdot H(t-\tau) \in \mathcal{L}_{\text {loc }}^{1}(\mathbb{R})$. Then

$$
D^{2}[\psi(t) \tilde{H}(t-\tau)]=\frac{d^{2}}{d t^{2}} \psi(t) \cdot \tilde{H}(t-\tau)+\psi^{\prime}(\tau) \delta(t-\tau)+\psi(\tau) D \delta(t-\tau)
$$

Corollary 2. Let $\tau \in \mathbb{R}, l \in \mathbb{Z}_{>0}, \psi(t)$ be such that $\frac{d^{l}}{d t^{l}} \psi(t) \cdot H(t-\tau) \in \mathcal{L}_{\text {loc }}^{1}(\mathbb{R})$, and $\tilde{\psi}_{\tau}(t)=\psi(t) \tilde{H}(t-$ $\tau)$. Then

$$
D^{l} \tilde{\psi}_{\tau}(t)=D^{l}[\psi(t) \tilde{H}(t-\tau)]=\frac{d^{l}}{d t^{l}} \psi(t) \cdot \tilde{H}(t-\tau)+\left\langle D^{l} \hat{\psi}_{\tau}(D)\right\rangle_{0} \delta(t-\tau),
$$

([5], Section 6.3), where

$$
\left\langle D^{l} \hat{\psi}_{\tau}(D)\right\rangle_{0}=\sum_{k=0}^{l-1} \psi^{(k)}(\tau) D^{l-k-1}
$$

When $l=1$ and $l=2$, we have

$$
\left\langle D \hat{\psi}_{\tau}(D)\right\rangle_{0}=\psi(\tau), \quad\left\langle D^{2} \hat{\psi}_{\tau}(D)\right\rangle_{0}=\psi^{\prime}(\tau)+\psi(\tau) D
$$

Equations (16) are obtained by comparing Equation (14) with Equations (12) and (13).

Corollary 3. Let $\tau \in \mathbb{R}, n \in \mathbb{Z}_{>0}, \psi(t)$ be such that $\frac{d^{n}}{d t^{n}} \psi(t) \cdot H(t-\tau) \in \mathcal{L}_{\text {loc }}^{1}(\mathbb{R}), \tilde{\psi}_{\tau}(t):=\psi(t) \tilde{H}(t-\tau)$, and

$$
p_{n}\left(t, \frac{d}{d t}\right) \psi(t):=\sum_{l=0}^{n} a_{l}(t) \frac{d^{l}}{d t^{l}} \psi(t)
$$

where $a_{l}(t)$ are polynomials of $t$. Then

$$
p_{n}(t, D) \tilde{\psi}_{\tau}(t):=\sum_{l=0}^{n} a_{l}(t) D^{l} \tilde{\psi}_{\tau}(t)=p_{n}\left(t, \frac{d}{d t}\right) \psi(t) \cdot \tilde{H}(t-\tau)+\sum_{l=1}^{n} a_{l}(t)\left\langle D^{l} \hat{\psi}_{\tau}(D)\right\rangle_{0} \delta(t-\tau),
$$

where $\left\langle D^{l} \hat{\psi}_{\tau}(D)\right\rangle_{0}$ are given by Equations (15) and (16).

In estimating the last term of Equation (18), we use the following lemma.

Lemma 5. Let $\tilde{u}(t) \in \mathcal{D}_{R}^{\prime}$, and $h(t)$ be such that $h(t) \phi(t) \in \mathcal{D}_{R}$ if $\phi \in \mathcal{D}_{R}$. Then

$$
h(t) D \tilde{u}(t)=D[h(t) \tilde{u}(t)]-h^{\prime}(t) \tilde{u}(t) .
$$

Proof.

$$
\langle h(t) D \tilde{u}(t), \phi(t)\rangle=\left\langle\tilde{u}(t), D_{W}[h(t) \phi(t)]\right\rangle=\left\langle-h^{\prime}(t) \tilde{u}(t)+D[h(t) \tilde{u}(t)], \phi(t)\right\rangle,
$$

since $D_{W}[h(t) \phi(t)]=-h^{\prime}(t) \phi(t)+h(t) D_{W} \phi(t)$. 


\subsection{Fractional Derivative and Distributions in the Space $\mathcal{D}_{R}^{\prime}$}

We consider the space $\mathcal{D}_{R}^{\prime}$ [11,12]. A regular distribution in $\mathcal{D}_{R}^{\prime}$ is such a distribution that it corresponds to a function which is locally integrable on $\mathbb{R}$ and has a support bounded on the left. A distribution $\tilde{h}$, which is not a regular one, is expressed as $\tilde{h}(t)=D^{n} \tilde{f}(t)$, by $n \in \mathbb{Z}_{>0}$ and a regular distribution $\tilde{f} \in \mathcal{D}_{R}^{\prime}$. The space $\mathcal{D}_{R}$, that is dual to $\mathcal{D}_{R}^{\prime}$, is the space of testing functions, which are infinitely differentiable on $\mathbb{R}$ and have a support bounded on the right.

We consider the Riemann-Liouville fractional integral and derivatives ${ }_{c} D_{R}^{\mu} f(z)$ of order $\mu \in \mathbb{C}$, when we may usually discuss the derivative $\frac{d^{n}}{d z^{n}} f(z)=f^{(n)}(z)$ of order $n \in \mathbb{Z}_{>0}$; see [16] and ([17], Section 2.3.2). In the following definition, $P(c, z)$ is the path from $c \in \mathbb{C}$ to $z \in \mathbb{C}$, and $\mathcal{L}^{1}(P(c, z))$ is the class of functions which are integrable on $P(c, z)$, and $\lfloor x\rfloor$ for $x \in \mathbb{R}$ denotes the greatest integer not exceeding $x$. In the following study, we choose the value $c=0$.

Definition 3. Let $c \in \mathbb{C}, z \in \mathbb{C}, f(\zeta) \in \mathcal{L}^{1}(P(c, z))$, and $f(\zeta)$ be continuous in a neighborhood of $\zeta=z$. Then the Riemann-Liouville fractional integral of order $\lambda \in{ }_{+} \mathbb{C}$ is defined by

$$
{ }_{c} D_{R}^{-\lambda} f(z)=\frac{1}{\Gamma(\lambda)} \int_{c}^{z}(z-\zeta)^{\lambda-1} f(\zeta) d \zeta,
$$

and the Riemann-Liouville fractional derivative of order $\mu \in \mathbb{C}$ satisfying $\operatorname{Re} \mu \geq 0$ is defined by

$$
{ }_{c} D_{R}^{\mu} f(z)={ }_{c} D_{R}^{m}\left[{ }_{c} D_{R}^{\mu-m} f(z)\right],
$$

when the rhs exists, where $m=\lfloor\operatorname{Re} \mu\rfloor+1$, and ${ }_{c} D_{R}^{m} f(z)=\frac{d^{m}}{d z^{m}} f(z)=f^{(m)}(z)$ for $m \in \mathbb{Z}_{>-1}$.

Definition 4. Let $\lambda \in{ }_{+} \mathbb{C}$, and $\tilde{f}$ be a regular distribution in $\mathcal{D}_{R^{\prime}}^{\prime}$, that corresponds to a function $f$. Then we have a regular distribution which corresponds to ${ }_{0} D_{R}^{-\lambda} f(t)$. We denote it $D^{-\lambda} \tilde{f}(t)$, and then

$$
\left\langle D^{-\lambda} \tilde{f}, \phi\right\rangle=\int_{-\infty}^{\infty}\left[{ }_{0} D_{R}^{-\lambda} f(t)\right] \phi(t) d t=\left\langle\tilde{f}, D_{W}^{-\lambda} \phi\right\rangle=\int_{-\infty}^{\infty} f(x)\left[D_{W}^{-\lambda} \phi(x)\right] d x,
$$

for every $\phi \in \mathcal{D}_{R}$. Then by using Equation (21) in the second member of Equation (23), we obtain

$$
D_{W}^{-\lambda} \phi(x)=\frac{1}{\Gamma(\lambda)} \int_{x}^{\infty}(t-x)^{\lambda-1} \phi(t) d t
$$

This formula shows that $D_{W}^{-\lambda} \phi$ does not belong to $\mathcal{D}$, even when $\phi \in \mathcal{D}$. As a consequence, the operator $D^{-\lambda}$ corresponding ${ }_{c} D_{R}^{-\lambda}$ cannot be an operator in the space $\mathcal{D}^{\prime}$, but we can confirm that it is an operator in the space $\mathcal{D}_{R}^{\prime}$.

Definition 5. Let $\tilde{h}(t) \in \mathcal{D}_{R}^{\prime}, m \in \mathbb{Z}_{>-1}, \lambda \in{ }_{+} \mathbb{C}, \mu=m-\lambda$ and $D^{\mu}=D^{m} D^{-\lambda}$. Then we have $D^{\mu} \tilde{h} \in \mathcal{D}_{R}^{\prime}$, which satisfies

$$
\left\langle D^{\mu} \tilde{h}, \phi\right\rangle=\left\langle\tilde{h}, D_{W}^{\mu} \phi\right\rangle,
$$

for every $\phi \in \mathcal{D}_{R}$, where $D_{W}^{\mu}=D_{W}^{-\lambda} D_{W}^{m}$.

In solving a differential equation, we assume that the solution $u(t)$ and the inhomogeneous part $f(t)$ for $t>0$, are expressed as a linear combination of

$$
g_{v}(t):=\frac{1}{\Gamma(v)} t^{v-1}, \quad v \in \mathbb{C} \backslash \mathbb{Z}_{<1}
$$


where $\Gamma(v)$ is the gamma function. The Laplace transform of $g_{v}(t)$ is given by $\mathcal{L}\left[g_{v}(t)\right]=s^{-v}$ if $v \in{ }_{+} \mathbb{C}$. We introduce the analytic continuation of the Laplace transform (AC-Laplace transform) of $g_{v}(t)$, which is expressed by $\mathcal{L}_{H}\left[g_{v}(t)\right]$, as in [1,2], such that

$$
\hat{g}_{v}(s)=\mathcal{L}_{H}\left[g_{v}(t)\right]=s^{-v}, \quad v \in \mathbb{C} \backslash \mathbb{Z}_{<1} .
$$

We often use the following formula [1,2].

Lemma 6. Let $v \in \mathbb{C} \backslash \mathbb{Z}_{<1}$ and $\mu \in \mathbb{C}$. Then for $t \in \mathbb{R}_{>0}$, we have

$$
{ }_{0} D_{R}^{\mu} g_{v}(t)= \begin{cases}g_{v-\mu}(t), & v-\mu \in \mathbb{C} \backslash \mathbb{Z}_{<1}, \\ 0, & v-\mu \in \mathbb{Z}_{<1} .\end{cases}
$$

Condition 1. A function of $t \in \mathbb{R}$ multiplied by $H(t)$, e.g. $u(t) H(t)$, is expressed as a linear combination of $g_{v}(t) H(t)$ for $v \in S$, where $S$ is an enumerable set of $v \in \mathbb{C} \backslash \mathbb{Z}_{<1}$ satisfying $\operatorname{Re} v>-M$ for some $M \in \mathbb{Z}_{>-1}$.

When $u(t)$ satisfies Condition 1 , it is expressed as follows:

$$
u(t)=\sum_{v \in S} u_{v-1} g_{v}(t)=\sum_{v \in S} u_{v-1} \frac{1}{\Gamma(v)} t^{v-1},
$$

where $u_{v-1} \in \mathbb{C}$ are constants. When $\hat{u}(s)=\mathcal{L}_{H}[u(t)]$ exists, it is expressed by

$$
\hat{u}(s)=\sum_{v \in S} u_{v-1} \hat{g}_{v}(s)=\sum_{v \in S} u_{v-1} s^{-v} .
$$

Definition 6. Let $g_{v}(t)$ and $\hat{g}_{v}(s)$ be given by Equations (26) and (27), respectively. Then $\tilde{g}_{v}(t) \in \mathcal{D}_{R}^{\prime}$ is defined by

$$
\tilde{g}_{v}(t)=\hat{g}_{v}(D) \delta(t)=D^{-v} \delta(t), \quad v \in \mathbb{C} \backslash \mathbb{Z}_{<1},
$$

and is denoted by $g_{v}(t) \tilde{H}(t)$.

Remark 1. When $v \in{ }_{+} \mathbb{C}$, we can confirm that $\tilde{g}_{v}(t)=g_{v}(t) \tilde{H}(t)$ is a regular distribution which corresponds to $g_{v}(t) H(t)$, as

$$
\begin{aligned}
\left\langle\tilde{g}_{v}, \phi\right\rangle & =\left\langle D^{-v} \delta(t), \phi(t)\right\rangle=\left\langle D^{-v+1} \tilde{H}(t), \phi(t)\right\rangle=\int_{-\infty}^{\infty} H(t)\left[D_{W}^{-v+1} \phi(t)\right] d t \\
& =\int_{-\infty}^{\infty}\left[{ }_{0} D_{R}^{-v+1} H(t)\right] \phi(t) d t=\int_{-\infty}^{\infty}\left[g_{v}(t) H(t)\right] \phi(t) d t
\end{aligned}
$$

with the aid of Equations (9), (11) and (23), Definition 5, and formulas $g_{1}(t)=1, g_{v}(t)={ }_{0} D_{R}^{-v+1} g_{1}(t)$ and $g_{1}(t)={ }_{0} D_{R}^{v-1} g_{v}(t)$ for $v \in \mathbb{C} \backslash \mathbb{Z}_{<1}$, which are obtained from Equations (26) and (28).

Lemma 7. Let $g_{v}(t), \hat{g}_{v}(s)$ and $\tilde{g}_{v}(t)$ be as in Definition 6. Then for $\mu \in \mathbb{C}$, we have

$$
D^{\mu} \tilde{g}_{v}(t)={ }_{0} D_{R}^{\mu} g_{v}(t) \cdot \tilde{H}(t)+\left\langle D^{\mu} \hat{g}_{v}(D)\right\rangle_{0} \delta(t),
$$

where

$$
\left\langle D^{\mu} \hat{g}_{v}(D)\right\rangle_{0}= \begin{cases}D^{m}, & m=\mu-v \in \mathbb{Z}_{>-1}, \\ 0, & \mu-v \notin \mathbb{Z}_{>-1} .\end{cases}
$$

Proof. By using Equations (28) and (31), we confirm Equation (32). 
Lemma 8. Let $u(t)$ and $\hat{u}(s)=\mathcal{L}_{H}[u(t)]$ be expressed by Equations (29) and (30), respectively. Then $\tilde{u}(t)=$ $u(t) \tilde{H}(t) \in \mathcal{D}_{R}^{\prime}$ is expressed as

$$
\tilde{u}(t)=\sum_{v \in S} u_{v-1} \tilde{g}_{v}(t)=\sum_{v \in S} u_{v-1} \hat{g}_{v}(D) \delta(t)=\hat{u}(D) \delta(t),
$$

in accordance with Definition 6.

Lemma 9. Let $l \in \mathbb{Z}_{>0}, u(t)$ be expressed by Equation (29), $\tilde{u}(t):=u(t) \tilde{H}(t)$, and $\hat{u}(s):=\mathcal{L}_{H}[u(t)]$. Then

$$
D^{l} \tilde{u}(t)=D^{l}[u(t) \tilde{H}(t)]=\frac{d^{l}}{d t} u(t) \cdot \tilde{H}(t)+\left\langle D^{l} \hat{u}(D)\right\rangle_{0} \delta(t),
$$

where

$$
\left\langle D^{l} \hat{u}(D)\right\rangle_{0}=\sum_{k=0}^{l-1} u_{k} D^{l-k-1}
$$

When $l=1,2$ and 3 , we have

$$
\langle D \hat{u}(D)\rangle_{0}=u_{0}, \quad\left\langle D^{2} \hat{u}(D)\right\rangle_{0}=u_{0} D+u_{1}, \quad\left\langle D^{3} \hat{u}(D)\right\rangle_{0}=u_{0} D^{2}+u_{1} D+u_{2} .
$$

Proof. By using Equation (34) for $\tilde{u}(t)$, and Lemma 7 for $\mu=l \in \mathbb{Z}_{>0}$, we have contributions to $\left\langle D^{l} \hat{u}(D)\right\rangle_{0} \delta(t)$ from the terms of $v-1=k \in \mathbb{Z}_{>-1}$ and $m=l-k-1 \in \mathbb{Z}_{>-1}$ in Equation (35).

Remark 2. Even when the series on the rhs of Equation (30) does not converge for any s, the series on the rhs of Equation (34) may converge in an interval of $t$ on $\mathbb{R}$. In such a case, we use $\hat{u}(s)$ to represent the series on the rhs of Equation (30).

\subsection{Fractional Derivative and Distributions in the Space $\mathcal{D}_{W}^{\prime}$}

We adopt the space $\mathcal{D}_{W}^{\prime}$ of distributions, such that regular ones correspond to functions which may increase slower than $e^{\epsilon t}$ for all $\epsilon \in \mathbb{R}_{>0}$ as $t \rightarrow \infty$. Then the dual space $\mathcal{D}_{W}$ consists of functions which are infinitely differentiable on $\mathbb{R}$ and decay more rapidly than $t^{-N}$ for all $N \in \mathbb{Z}_{>0}$ as $t \rightarrow \infty$.

Remark 3. In the book of Miller and Ross ([18], Chapter VII), one chapter is used to discuss the Weyl fractional integral and differentiation in the space $\mathcal{D}_{W}$, where notation $W^{\mu} g(t)$ is used in place of $D_{W}^{\mu} g(t)$ for $\mu \in \mathbb{R}$ and $\phi \in \mathcal{D}_{W}$.

Lemma 10. Let $m \in \mathbb{Z}_{>-1}, \lambda \in{ }_{+} \mathbb{C}$ and $\mu=m-\lambda$. Then

$$
D_{W}^{m} e^{-s t}=s^{m} e^{-s t}, \quad D_{W}^{-\lambda} e^{-s t}=s^{-\lambda} e^{-s t}, \quad D_{W}^{\mu} e^{-s t}=s^{\mu} e^{-s t} .
$$

Proof. The second equation is confirmed with the aid of Equation (24). The third equation is confirmed with the aid of Definition 5, which states $D_{W}^{\mu}=D_{W}^{-\lambda} D_{W}^{m}$.

Lemma 11. Let $\tilde{u}(t)=u(t) \tilde{H}(t)$ be a regular distribution in $\mathcal{D}_{W}^{\prime}$, that corresponds to function $u(t) H(t)$, $\hat{u}(s)=\mathcal{L}[u(t)]=\int_{0}^{\infty} u(t) e^{-s t} d t$, and $\tilde{w}(t)$ and $\hat{w}(s)$ be given by $\tilde{w}(t)=D^{l} \tilde{u}(t)$ and $\hat{w}(s)=s^{l} \hat{u}(s)$ for $l \in \mathbb{Z}_{>0}$. Then for $\mu \in \mathbb{C}$, we have

$$
\text { (i) }\left\langle\tilde{u}(t), e^{-s t}\right\rangle=\hat{u}(s), \quad(\text { ii })\left\langle D^{\mu} \tilde{u}(t), e^{-s t}\right\rangle=s^{\mu} \hat{u}(s), \quad \text { (iii) }\left\langle t D^{\mu} \tilde{u}(t), e^{-s t}\right\rangle=-\frac{d}{d s}\left[s^{\mu} \hat{u}(s)\right],
$$

and also these equations with $\tilde{u}(t)$ and $\hat{u}(s)$ replaced by $\tilde{w}(t)$ and $\hat{w}(s)$, respectively. 
Proof. The lhs of these equations are expressed as follows: (i) $\left\langle\tilde{u}(t), e^{-s t}\right\rangle=\int_{-\infty}^{\infty} u(t) H(t) e^{-s t} d t=$ $\mathcal{L}[u(t)]=\hat{u}(s)$, (ii) $\left\langle D^{\mu} \tilde{u}(t), e^{-s t}\right\rangle=\left\langle\tilde{u}(t), D_{W}^{\mu} e^{-s t}\right\rangle=s^{\mu}\left\langle\tilde{u}(t), e^{-s t}\right\rangle=s^{\mu} \hat{u}(s)$, and

(iii) $\left\langle t D^{\mu} \tilde{u}(t), e^{-s t}\right\rangle=\left\langle D^{\mu} \tilde{u}(t), t e^{-s t}\right\rangle=-\frac{d}{d s}\left\langle D^{\mu} \tilde{u}(t), e^{-s t}\right\rangle=-\frac{d}{d s}\left[s^{\mu} \hat{u}(s)\right]$.

This lemma shows that the formulas in Equation (39) are valid for all distributions $\tilde{u}(t) \in \mathcal{D}_{W}^{\prime}$.

Corollary 4. Let the condition of Lemma 6 be satisfied. Then $\delta(t) \in \mathcal{D}_{W}^{\prime}, \tilde{g}_{v}(t) \in \mathcal{D}_{W}^{\prime}$ for $v \in \mathbb{C} \backslash \mathbb{Z}_{<1}$, and

$$
\begin{gathered}
\left\langle\tilde{H}(t), e^{-s t}\right\rangle=\frac{1}{s} ;\left\langle\delta(t), e^{-s t}\right\rangle=1 ; \\
\left\langle\tilde{g}_{v}(t), e^{-s t}\right\rangle=\left\langle\hat{g}_{v}(D) \delta(t), e^{-s t}\right\rangle=\hat{g}_{v}(s)=s^{-v} .
\end{gathered}
$$

Proof. $\left\langle\delta(t), e^{-s t}\right\rangle=\left\langle D \tilde{H}(t), e^{-s t}\right\rangle=\left\langle\tilde{H}(t), D_{W} e^{-s t}\right\rangle=s^{-1} s=1$.

Corollary 5. Let $\tilde{u}(t) \in \mathcal{D}_{W}^{\prime}$ be expressed as $\tilde{u}(t)=\hat{u}(D) \delta(t)$. Then

$$
\left\langle\tilde{u}(t), e^{-s t}\right\rangle=\left\langle\hat{u}(D) \delta(t), e^{-s t}\right\rangle=\hat{u}(s) .
$$

Lemma 12. Let $l \in \mathbb{Z}_{>0}, u(t)$ be expressed by Equation (29), and $\hat{u}(s):=\mathcal{L}_{H}[u(t)]$. Then

$$
s^{l} \hat{u}(s)=\mathcal{L}_{H}\left[\frac{d^{l}}{d t^{l}} u(t)\right]+\left\langle s^{l} \hat{u}(s)\right\rangle_{0},
$$

where $\left\langle s^{l} \hat{u}(s)\right\rangle_{0}$ are given by Equations (36) and (37) with $D$ replaced by $s$.

Proof. We put $\tilde{u}(t)=u(t) \tilde{H}(t)$, and then we have Equation (35). Applying Lemma 11 and Corollary 5 to Equation (35), we obtain Equation (42).

Remark 4. In the book by Zemanian ([5], Section 8.3), he discussed the Laplace transform by adopting the space $\mathcal{D}_{L}^{\prime}$ of distributions, such that regular ones correspond to functions which may increase slower than $e^{(\lambda+\epsilon) t}$ for all $\epsilon \in \mathbb{R}_{>0}$ as $t \rightarrow \infty$, for a fixed $\lambda \in \mathbb{R}_{>0}$. Then the dual space $\mathcal{D}_{L}$ consists of functions which are infinitely differentiable on $\mathbb{R}$ and decay more rapidly than $e^{-\lambda t} t^{-N}$ for all $N \in \mathbb{Z}_{>0}$ as $t \rightarrow \infty$. Then the above formulas in the present section are valid when $\operatorname{Re} s>\lambda$. We here adopt the case when $\lambda=0$.

Remark 5. Let $u(t)$ and $\tilde{u}(t)$ be expressed by Equations (29) and (34), respectively. Then $\tilde{u}(t)$ does not usually belongs to $\mathcal{D}_{W}^{\prime}$. Even in that case, we use the following equation:

$$
\left\langle\tilde{u}(t), e^{-s t}\right\rangle=\left\langle\hat{u}(D) \delta(t), e^{-s t}\right\rangle=\sum_{v \in S} u_{v-1}\left\langle\tilde{g}_{v}(t), e^{-s t}\right\rangle=\sum_{v \in S} u_{v-1} \hat{g}_{v}(s)=\hat{u}(s) .
$$

\subsection{Some Primitive Leibniz's formulas}

Lemma 13. Let $\tilde{u}(t)=\hat{u}(D) \delta(t) \in \mathcal{D}_{R}^{\prime}$, and $\mu \in \mathbb{C}$. Then we have two special ones of Leibniz's formula:

$$
\begin{aligned}
D^{\mu}[t \tilde{u}(t)] & =t D^{\mu} \tilde{u}(t)+\mu D^{\mu-1} \tilde{u}(t)=(t D+\mu) D^{\mu-1} \tilde{u}(t), \\
D^{\mu}\left[t^{2} \tilde{u}(t)\right] & =t^{2} D^{\mu} \tilde{u}(t)+2 \mu t D^{\mu-1} \tilde{u}(t)+\mu(\mu-1) D^{\mu-2} \tilde{u}(t) \\
& =\left(t^{2} D^{2}+2 \mu t D+\mu(\mu-1)\right) D^{\mu-2} \tilde{u}(t) .
\end{aligned}
$$

Proof. Lemma 11 shows that when Equation (44) is satisfied, we have

$$
s^{\mu}\left[-\frac{d}{d s} \hat{u}(s)\right]=-\frac{d}{d s}\left[s^{\mu} \hat{u}(s)\right]+\mu s^{\mu-1} \hat{u}(s),
$$

which is confirmed since $-\frac{d}{d s}\left[s^{\mu} \hat{u}(s)\right]=-\mu s^{\mu-1} \hat{u}(s)-s^{\mu} \frac{d}{d s} \hat{u}(s)$. Formula (45) is obtained with the aid of Equation (44). 


\section{Green's Function for Inhomogeneous Differential Equations with Polynomial Coefficients}

In Sections 4 and 5 , we study the solution $u(t)$ of inhomogeneous differential equations with polynomial coefficients:

$$
p_{n}\left(t, \frac{d}{d t}\right) u(t):=\sum_{l=0}^{n} a_{l}(t) \frac{d^{l}}{d t} u(t)=f(t), \quad t>0,
$$

where $n \in \mathbb{Z}_{>0}$, and $a_{l}(t)$ are polynomials of $t$ satisfying $a_{0}(t) \neq 0$ and $a_{n}(t) \neq 0$.

For the inhomogeneous term $f(t)$, we consider the following three cases.

\section{Condition 2.}

(i) $f(t)$ is a locally integrable function on $\mathbb{R}_{\geq 0}$,

(ii) $f(t)={ }_{0} D_{R}^{\beta} f_{\beta}(t)$ satisfies Condition 1 , where $f_{\beta}(t)$ is a locally integrable function on $\mathbb{R}_{\geq 0}$,

(iii) $f(t)=g_{v}(t)=\frac{1}{\Gamma(v)} t^{v-1}$, and $\tilde{f}(t)=f(t) \tilde{H}(t)=\hat{f}(D) \delta(t)=\hat{g}_{v}(D) \delta(t)=D^{-v} \delta(t)$, for $v \in \mathbb{C} \backslash \mathbb{Z}_{<1}$.

Lemma 14. Let $u(t)$ be a solution of Equation (47), which is expressed by Equation (29). Then the differential equation satisfied by distribution $\tilde{u}(t):=u(t) \tilde{H}(t)$ is

$$
\begin{aligned}
p_{n}(t, D) \tilde{u}(t): & =\sum_{l=0}^{n} a_{l}(t) D^{l} \tilde{u}(t)=p_{n}\left(t, \frac{d}{d t}\right) u(t) \cdot \tilde{H}(t)+\sum_{l=1}^{n} a_{l}(t)\left\langle D^{l} \hat{u}(D)\right\rangle_{0} \delta(t) \\
& =\tilde{f}(t)+\sum_{l=1}^{n} a_{l}(t)\left\langle D^{l} \hat{u}(D)\right\rangle \delta(t),
\end{aligned}
$$

where $\tilde{f}(t)=f(t) \tilde{H}(t)$, and $\left\langle D^{l} \hat{u}(D)\right\rangle_{0}$ are given by Equations (36) and (37).

Proof. Equation (48) is obtained by using Equation (35).

Lemma 15. Let Condition 2(i) be satisfied. Then $\tilde{f}(t)=f(t) \tilde{H}(t)$, which corresponds to $f(t) H(t)$, is expressed as

$$
\tilde{f}(t)=\int_{0}^{\infty} \delta(t-\tau) f(\tau) d \tau
$$

Proof. The rhs is equal to the lhs, since

$$
\begin{aligned}
& \left\langle\int_{0}^{\infty} \delta(t-\tau) f(\tau) d \tau, \phi(t)\right\rangle=-\int_{-\infty}^{\infty}\left[\int_{0}^{\infty} H(t-\tau) f(\tau) d \tau\right] \phi^{\prime}(t) d t \\
& \quad=-\int_{0}^{\infty} f(\tau)\left[\int_{-\infty}^{\infty} H(t-\tau) \phi^{\prime}(t) d t\right] d \tau=\int_{-\infty}^{\infty} f(\tau) H(\tau) \phi(\tau) d \tau=\langle\tilde{f}, \phi\rangle,
\end{aligned}
$$

where $\phi \in \mathcal{D}_{R}$.

Definition 7. For Equation (47), the Green's function $G(t, \tau)$ for $\tau \in \mathbb{R}_{\geq 0}$ is such that $G(t, \tau)=G(t, \tau) H(t-\tau)$, and $\tilde{G}(t, \tau)=G(t, \tau) \tilde{H}(t-\tau)$ satisfies

$$
p_{n}(t, D) \tilde{G}(t, \tau)=\delta(t-\tau)
$$

Lemma 16. The Green's function $G(t, \tau)$ for Equation (47) satisfies

$$
p_{n}\left(t, \frac{d}{d t}\right) G(t, \tau)=\sum_{l=0}^{n} a_{l}(t) \frac{d^{l}}{d t^{l}} G(t, \tau)=0, \quad t>\tau,
$$


and $G(t, \tau)=0$ for $t<\tau$. The values of $G(t, \tau)$ and its derivatives at $t=\tau$ are determined by

$$
\sum_{l=1}^{n} a_{l}(t)\left\langle D^{l} \hat{G}(D, \tau)\right\rangle_{0} \delta(t-\tau)=\delta(t-\tau),
$$

where $\left\langle D^{l} \hat{G}(D, \tau)\right\rangle_{0}$ is given by Equations (15) and (16) with $\hat{\psi}_{\tau}(D)$ and $\tilde{\psi}_{\tau}(t)$ replaced by $\hat{G}(D, \tau)$ and $\tilde{G}(t, \tau)$, respectively.

Proof. This is confirmed by comparing Equation (50) with Equation (18), where $\psi(t), \hat{\psi}_{\tau}(D)$ and $\tilde{\psi}_{\tau}(t)$ are replaced by $G(t, \tau), \hat{G}(D, \tau)$ and $\tilde{G}(t, \tau)$, respectively.

Lemma 17. $\tilde{u}_{f}(t)$ given by

$$
\tilde{u}_{f}(t)=\int_{0}^{\infty} \tilde{G}(t, \tau) f(\tau) d \tau
$$

is a particular solution of Equation (48) for the term $\tilde{f}(t)$.

Proof. By using the rhs of Equation (53) in the lhs of Equation (48), we obtain $f(t) \tilde{H}(t)$ by using Equations (50) and (49).

Lemma 18. Let $G(t, \tau)$ be defined by Definition 7 for Equation (47), Condition 2(i) be satisfied, and $u_{f}(t)$ be given by

$$
u_{f}(t)=\int_{0}^{t} G(t, \tau) f(\tau) d \tau
$$

Then $u_{f}(t)$ and $\tilde{u}_{f}(t)=u_{f}(t) \tilde{H}(t)$ are particular solutions of Equations (47) and (48), respectively.

Proof. We show that when $\tilde{u}_{f}(t)$ and $u_{f}(t)$ are defined by Equations (53) and (54), respectively, $\tilde{u}_{f}(t)=u_{f}(t) \tilde{H}(t)$. This is confirmed as follows:

$$
\begin{aligned}
\left\langle\tilde{u}_{f}(t), \phi\right\rangle & =\int_{0}^{\infty}\left[\int_{-\infty}^{\infty} G(t, \tau) H(t-\tau) \phi(t) d t\right] f(\tau) d \tau \\
& =\int_{-\infty}^{\infty}\left[\int_{0}^{t} G(t, \tau) f(\tau) d \tau\right] H(t) \phi(t) d t=\left\langle u_{f}(t) \tilde{H}(t), \phi(t)\right\rangle,
\end{aligned}
$$

where $\phi \in \mathcal{D}_{R}$. The proof of the lemma is completed with the aid of Lemmas 17 and 14 .

Green's Function for Inhomogeneous Differential Equations with Constant Coefficients

We now consider the case when $a_{l}(t)$ do not depend on $t$, and hence in place of Equation (47), we have

$$
p_{n}\left(\frac{d}{d t}\right) u(t):=\sum_{l=0}^{n} a_{l} \frac{d^{l}}{d t^{l}} u(t)=f(t), \quad t>0,
$$

where $n \in \mathbb{Z}_{>0}$, and $a_{l} \in \mathbb{C}$ are constants satisfying $a_{0} \neq 0$ and $a_{n} \neq 0$. When we use the formulas in the preceding section, we assume that $a_{l}(t)$ and $p_{n}\left(t, \frac{d}{d t}\right)$ represent $a_{l}$ and $p_{n}\left(\frac{d}{d t}\right)$, respectively. In place of Equation (48), we have

$$
p_{n}(D) \tilde{u}(t):=\sum_{l=0}^{n} a_{l} D^{l} \tilde{u}(t)=\tilde{f}(t)+\sum_{l=1}^{n} a_{l}\left\langle D^{l} \hat{u}(D)\right\rangle_{0} \delta(t),
$$

where $\tilde{f}(t)=f(t) \tilde{H}(t)=\hat{f}(D) \delta(t)$, and $\left\langle D^{l} \hat{u}(D)\right\rangle_{0}$ are given by Equations (36) and (37). 
We denote the solution $G(t, 0)$ obtained by Definition 7 , by $G(t)$. Then $\tilde{G}(t)=G(t) \tilde{H}(t)$ satisfies

$$
p_{n}(D) \tilde{G}(t)=\delta(t)
$$

By using this equation, we confirm the following lemma.

Lemma 19. $\tilde{u}(t)$ given by

$$
\tilde{u}(t)=\hat{f}(D) \tilde{G}(t)+\sum_{l=1}^{n} a_{l}\left\langle D^{l} \hat{u}(D)\right\rangle_{0} \tilde{G}(t),
$$

is a solution of Equation (57).

Proof. Putting $\tilde{u}(t)$ given by Equation (59) on the lhs of Equation (57) and using Equation (58), we confirm that Equation (57) is satisfied.

By Lemma 16, we have

Lemma 20. Let $G(t)$ be defined by

$$
p_{n}\left(\frac{d}{d t}\right) G(t):=\sum_{l=0}^{n} a_{l} \frac{d^{l}}{d t^{l}} G(t)=\frac{d}{d t} H(t)=0, \quad t>0,
$$

and the initial values of $G(t)$ and its derivatives satisfy $\sum_{l=1}^{n} a_{l}\left\langle D^{l} \hat{G}(D)\right\rangle_{0}=1$, so that

$$
a_{n} G^{(n-1)}(0)=H(0)=1 ; \quad G^{(l)}(0)=0, \quad\left(l \in \mathbb{Z}_{[0, n-2]}, n \in \mathbb{Z}_{>1}\right) .
$$

Then $\tilde{G}(t)=G(t) \tilde{H}(t)$ satisfies (58), and $\tilde{G}(t, \tau)=G(t-\tau) H(t-\tau)$ for $\tau \geq 0$ satisfies Definition 7 if $p_{n}(t, D)=p_{n}(D)$.

Proof. By comparing Equations (50) and (58), we confirm the last statement.

Lemma 21. Let Condition 2(i) be satisfied, and $u(t)$ be given by

$$
u(t)=u_{f}(t)+\sum_{l=1}^{n} a_{l}\left\langle\frac{d^{l}}{d t^{l}} \hat{u}\left(\frac{d}{d t}\right)\right\rangle_{0} G(t),
$$

where

$$
u_{f}(t)=\int_{0}^{t} G(t-\tau) f(\tau) d \tau
$$

Then $u(t)$ and $\tilde{u}(t)=u_{f}(t) \tilde{H}(t)$ are solutions of Equations (56) and (57), respectively.

Proof. Equation (63) follows from Lemma 18.

Remark 6. In ([5], Section 6.3), the solution of an inhomogeneous differential equation with constant coefficients is discussed, where the formulas in Corollary 2, Formulas (60) and (61) for the Green's function and Formula (62) for the solution, are presented.

\section{Particular Solution of Kummer's Differential Equation}

Kummer's differential equation with an inhomogeneous term $f(t)$ is given in Equation (2). If $f(t)=0$ and $c \notin \mathbb{Z}$, the basic solutions $K_{1}(t)$ and $K_{2}(t)$ of Equation (2) are given by Equations (3) and (4). 
We now obtain a particular solution of this equation by the method stated in Section 3.

\subsection{Green's Function for Kummer's Differential Equation in which Condition 2(i) Is Satisfied}

The following two lemmas are proved in Section 4.4.

Lemma 22. Let $u(t)$ be the solution of Equation (2), which is expressed by Equation (29). Then the differential equation satisfied by $\tilde{u}(t)=u(t) \tilde{H}(t)$ is given by

$$
p_{K}(t, D) \tilde{u}(t)=f(t) \tilde{H}(t)+u_{0}(c-1) \delta(t) .
$$

Lemma 23. Let $K_{1}(t)$ and $K_{2}(t)$ be given by Equations (3) and (4), and $\psi_{K}(t, \tau)$ for fixed $\tau>0$ be given by

$$
\psi_{K}(t, \tau):=c_{1}(\tau) \cdot K_{1}(t)+c_{2}(\tau) \cdot K_{2}(t),
$$

where $c_{1}(\tau)$ and $c_{2}(\tau)$ are constants which are so chosen that $\psi_{K}(t, \tau)=0$ when $t=\tau$. Then $G_{K}(t, 0)$ and $G_{K}(t, \tau)$ given by

$$
G_{K}(t, 0)=\frac{1}{c-1} \cdot K_{1}(t) H(t), \quad G_{K}(t, \tau)=\frac{1}{\tau \psi_{K}^{\prime}(\tau, \tau)} \psi_{K}(t, \tau) H(t-\tau),
$$

are the Green's functions for Equation (2), so that $\tilde{G}_{K}(t, 0)=G_{K}(t, 0) \tilde{H}(t)$ and $\tilde{G}_{K}(t, \tau)=G_{K}(t, \tau) \tilde{H}(t-\tau)$ satisfy

$$
p_{K}(t, D) \tilde{G}_{K}(t, 0)=\delta(t), \quad p_{K}(t, D) \tilde{G}_{K}(t, \tau)=\delta(t-\tau)
$$

Theorem 1. Let $G_{K}(t, \tau)$ and $\psi_{K}(t, \tau)$ be those given in Lemma 23, Condition 2(i) be satisfied, and $u_{f}(t)$ be given by

$$
u_{f}(t)=\int_{0}^{t} G_{K}(t, \tau) f(\tau) d \tau=\int_{0}^{t} \psi_{K}(t, \tau) \frac{f(\tau)}{\tau \psi_{K}^{\prime}(\tau, \tau)} d \tau
$$

Then $u_{f}(t)$ and $u_{f}(t) \tilde{H}(t)$ are particular solutions of Equations (2) and (64) for the terms $f(t)$ and $f(t) \tilde{H}(t)$, respectively.

Proof. This is confirmed with the aid of Lemma 18.

Remark 7. By using the first equation in Equation (67), we see that the particular solution of Equation (64) for the last term, is

$$
\tilde{u}_{1}(t)=u_{0}(c-1) \tilde{G}_{K}(t, 0)=u_{0} \cdot K_{1}(t) \tilde{H}(t) .
$$

The corresponding particular solution of Equation (2) is

$$
u_{1}(t)=u_{0}(c-1) G_{K}(t, 0)=u_{0} \cdot K_{1}(t) .
$$

Considering that the basic complementary solutions of Equation (2) are given by Equations (3) and (4), the general solution of Equation (2) is now given by

$$
u(t)=u_{f}(t)+u_{0} \cdot K_{1}(t)+c_{3} \cdot K_{2}(t) .
$$


The condition $\psi_{K}(\tau, \tau)=0$ requires that $c_{1}(\tau) \cdot K_{1}(\tau)=-c_{2}(\tau) \cdot K_{2}(\tau)$, and hence we may choose $\psi_{K}(t, \tau)$ as

$$
\psi_{K}(t, \tau)= \begin{cases}K_{1}(t)-\frac{K_{1}(\tau)}{K_{2}(\tau)} \cdot K_{2}(t), & \left|K_{1}(\tau)\right|<\left|K_{2}(\tau)\right|, \\ \frac{K_{2}(\tau)}{K_{1}(\tau)} \cdot K_{1}(t)-K_{2}(t), & \left|K_{1}(\tau)\right| \geq\left|K_{2}(\tau)\right| .\end{cases}
$$

\subsection{Green's Function for Kummer's Differential Equation in which Condition 2(ii) Is Satisfied}

We give the solution of Equation (2) of which the inhomogeneous term $f(t)$ satisfies Condition 2(ii), so that $\tilde{f}(t)=D^{\beta} \tilde{f}_{\beta}(t)$, and $f_{\beta}(t)$ satisfies Condition 2(i).

The following lemma is proved in Section 4.4.

Lemma 24. Let $\tilde{u}(t)$ be a solution of Equation (64), and $p_{\tilde{K}}(t, D)$ be related with $p_{K}(t, D)$ given by Equation (1), by

$$
p_{\tilde{K}}(t, D)=D^{-\beta} p_{K}(t, D) D^{\beta} .
$$

Then

$$
p_{\tilde{K}}(t, D):=t \cdot D^{2}+(c-\beta-b t) D-(a-\beta) b,
$$

and $\tilde{w}(t)=D^{-\beta} \tilde{u}(t)$ satisfies

$$
p_{\tilde{K}}(t, D) \tilde{w}(t)=f_{\beta}(t) \tilde{H}(t)+u_{0}(c-1) D^{-\beta} \delta(t) .
$$

Theorem 1 shows that the particular solution of Equation (75) for the term $f_{\beta}(t) \tilde{H}(t)$ is expressed by a particular solution of

$$
p_{\tilde{K}}\left(t, \frac{d}{d t}\right) w(t)=f_{\beta}(t), \quad t>0 .
$$

That solution is used in giving a solution of Equation (2) in Theorem 2 given below.

Remark 8. We note that $p_{\tilde{K}}(t, D)$ given by Equation (74) is obtained from $p_{K}(t, D)$ given by Equation (1), by replacing $a$ and $c$ by $a-\beta$ and $c-\beta$, respectively, and hence the complementary solutions $K_{\beta, 1}(t)$ and $K_{\beta, 2}(t)$ and Green's functions $G_{\tilde{K}}(t, 0)$ and $G_{\tilde{K}}(t, \tau)$ of Equation (76) are obtained from those $K_{1}(t), K_{2}(t), G_{K}(t, 0)$ and $G_{K}(t, \tau)$, respectively, of Equation (2), by the same replacement.

Now in place of Theorem 1, we have the following theorem, whose proof is given in Section 4.4.

Theorem 2. Let $G_{\tilde{K}}(t, \tau)$ and $\psi_{\tilde{K}}(t, \tau)$ be obtained from $G_{K}(t, \tau)$ and $\psi_{K}(t, \tau)$ by the replacement stated in Remark 8, Condition 2(ii) be satisfied, and $w_{g}(t)$ be given by

$$
w_{g}(t)=\int_{0}^{t} G_{\tilde{K}}(t, \tau) f_{\beta}(\tau) d \tau=\int_{0}^{t} \psi_{\tilde{K}}(t, \tau) \frac{f_{\beta}(\tau)}{\tau \psi_{\tilde{K}}^{\prime}(\tau, \tau)} d \tau .
$$

Then $u_{f}(t):={ }_{0} D_{R}^{\beta} w_{g}(t)$ and $u_{f}(t) \tilde{H}(t)$ are particular solutions of Equations (2) and (64) for the terms $f(t)$ and $f(t) \tilde{H}(t)$, respectively.

\subsection{Particular Solution of Kummer's Differential Equation in which Condition 2(iii) Is Satisfied}

We give the solution of Equation (2) of which the inhomogeneous term $f(t)$ satisfies Condition 2(iii), so that $f(t)=g_{v}(t)=\frac{1}{\Gamma(v)} t^{\nu-1}$ and $\tilde{f}(t)=f(t) \tilde{H}(t)=\hat{f}(D) \delta(t)=\hat{g}_{v}(D) \delta(t)=$ $D^{-v} \delta(t)$. Here we use $\beta \notin \mathbb{Z}_{>-1}$ in place of $-v$. 
Lemma 25. Let $\tilde{f}(t)=D^{\beta} \delta(t), p_{\tilde{K}}(t, D)$ be given by Equation $(74)$, and $\tilde{G}_{\tilde{K}}(t, 0)$ satisfy

$$
p_{\tilde{K}}(t, D) \tilde{G}_{\tilde{K}}(t, 0)=\delta(t) .
$$

Then the particular solution of Equation (64) for the term $f(t) \tilde{H}(t)=D^{\beta} \delta(t)$ is given by

$$
\tilde{u}_{f}(t)=D^{\beta} \tilde{G}_{\tilde{K}}(t, 0) .
$$

Proof. $\tilde{u}_{f}(t)$ satisfies $p_{K}(t, D) \tilde{u}_{f}(t)=D^{\beta} \delta(t)$ and hence $p_{\tilde{K}}(t, D) D^{-\beta} \tilde{u}_{f}(t)=\delta(t)$ by Equation (73). Comparing this with Equation (78), we see that Equation (79) is satisfied.

By Remark 8, with the aid of $G_{K}(t, 0)$ given by Equations (66) and (3), we have

Lemma 26. As the solution of Equation (78), we obtain

$$
\tilde{G}_{\tilde{K}}(t, 0)=G_{\tilde{K}}(t, 0) \tilde{H}(t), \quad G_{\tilde{K}}(t, 0)=\frac{1}{c-\beta-1} \cdot{ }_{1} F_{1}(a-\beta ; c-\beta ; b t) .
$$

Theorem 3. Let Condition 2(iii) be satisfied, and $G_{\tilde{K}}(t, 0)$ be given by Equation (80). Then the particular solution of Equation (2) is given by

$$
u_{f}(t)={ }_{0} D_{R}^{\beta} G_{\tilde{K}}(t, 0)=\frac{t^{-\beta}}{(c-\beta-1) \Gamma(1-\beta)} \cdot{ }_{2} F_{2}(1, a-\beta ; 1-\beta, c-\beta ; b t),
$$

where ${ }_{2} F_{2}\left(a_{1}, a_{2} ; c_{1}, c_{2} ; z\right)=\sum_{k=0}^{\infty} \frac{\left(a_{1}\right)_{k}\left(a_{2}\right)_{k}}{k !\left(c_{1}\right)_{k}\left(c_{2}\right)_{k}} z^{k}$.

Proof. $\tilde{u}_{f}(t)$ is given by Equations (79) and (80). Lemma 7 shows that $\tilde{u}_{f}(t)=u_{f}(t) \tilde{H}(t)$ if $u_{f}(t)$ is given by Equation (81), since $\beta \notin \mathbb{Z}_{>-1}$.

\subsection{Proofs of Lemmas 22-24 and Theorem 2}

Proof of Lemma 22. Equation (64) is a special one of (48), where $p_{n}, n, a_{2}(t), a_{1}(t)$ and $a_{0}(t)$ stand for $p_{K}, 2, t, c-b t$ and $-a b$, respectively. Hence by using Equation (37), we see that the second term on the rhs of Equation (48) becomes

$$
t\left(u_{0} D+u_{1}\right) \delta(t)+(c-b t) u_{0} \delta(t)=u_{0}(D[t \delta(t)]-\delta(t))+c u_{0} \delta(t)=u_{0}(c-1) \delta(t) .
$$

We prepare a lemma before proving Lemma 23.

Lemma 27. Let $\tau \geq 0, p_{K}\left(t, \frac{d}{d t}\right) \psi(t) \cdot H(t-\tau) \in \mathcal{L}_{l o c}^{1}$ and $\tilde{\psi}_{\tau}(t)=\psi(t) \tilde{H}(t-\tau)$. Then

$$
p_{K}(t, D) \tilde{\psi}_{\tau}(t)=p_{K}\left(t, \frac{d}{d t}\right) \psi(t) \cdot \tilde{H}(t-\tau)+\sum_{l=1}^{2} a_{l}(t)\left\langle D^{l} \hat{\psi}_{\tau}(D)\right\rangle_{0} \delta(t-\tau),
$$

where $a_{2}(t)=t, a_{1}(t)=c-b t, a_{0}(t)=a b$, and

$$
\sum_{l=1}^{2} a_{l}(t)\left\langle D^{l} \hat{\psi}_{\tau}(D)\right\rangle_{0} \delta(t-\tau)=\tau \psi^{\prime}(\tau) \delta(t-\tau)+\psi(\tau)(c-b \tau-1) \delta(t-\tau)+\psi(\tau) \tau D \delta(t-\tau) .
$$


Proof. Equation (83) is a special one of Equation (18) in Corollary 3. The lhs of Equation (84) is evaluated with the aid of Equation (16) as follows:

$$
\begin{aligned}
\sum_{l=1}^{2} a_{l}(t)\left\langle D^{l} \hat{\psi}_{\tau}(D)\right\rangle_{0} \delta(t-\tau) & =t\left[\psi^{\prime}(\tau)+\psi(\tau) D\right] \delta(t-\tau)+(c-b t) \psi(\tau) \delta(t-\tau) \\
& =\left[t \psi^{\prime}(\tau)+\psi(\tau)(c-b t)\right] \delta(t-\tau)+\psi(\tau) t D \delta(t-\tau) \\
& =\tau \psi^{\prime}(\tau) \delta(t-\tau)+\psi(\tau)(c-b \tau-1) \delta(t-\tau)+\psi(\tau) \tau D \delta(t-\tau),
\end{aligned}
$$

where Formula (19) with $h(t)$ and $\tilde{u}(t)$ replaced by $t$ and $\delta(t-\tau)$, respectively, is used in the last term of the third member.

Proof of Lemma 23. If we put $\psi(t)=\frac{1}{c-1} \cdot K_{1}(t)$ and $\tau=0$ in Equation (83), then we have $\tilde{\psi}_{\tau}(t)=\tilde{G}(t, 0)$ on the lhs, and the rhs is $\psi(0)(c-1) \delta(t)=\delta(t)$, since $K_{1}(0)=1$. If we put $\psi(t)=\frac{1}{\tau \psi_{K}^{\prime}(\tau, \tau)} \psi_{K}(t, \tau)$ and $\psi(\tau)=0$ in Equation (83), then we have $\tilde{\psi}_{\tau}(t)=\tilde{G}(t, \tau)$ on the lhs, and the rhs is $\tau \psi^{\prime}(\tau) \delta(t-\tau)=\delta(t-\tau)$.

Proof of Lemma 24. With the aid of Equation (44), we obtain

$$
\begin{aligned}
D^{-\beta}\left[p_{K}(t, D)\left[D^{\beta} \tilde{w}(t)\right]\right] & =D^{-\beta}\left[t \cdot D^{2}+(c-b t) \cdot D-a b\right]\left[D^{\beta} \tilde{w}(t)\right] \\
& =[(t \cdot D-\beta) D+c \cdot D-b(t \cdot D-\beta)-a b] \tilde{w}(t) \\
& =\left[t \cdot D^{2}+(c-\beta-b t) \cdot D-(a-\beta) b\right] \tilde{w}(t)=p_{\tilde{K}}(t, D) \tilde{w}(t),
\end{aligned}
$$

which gives Equation (73). When $\tilde{u}(t)=D^{\beta} \tilde{w}(t)$, Equation (64) shows that the lhs of Equation (85) is equal to $D^{-\beta}\left[\tilde{f}(t)+u_{0}(c-1) \delta(t)\right]$ and hence Equation (85) gives Equation (75).

Proof of Theorem 2. Theorem 1 states that when $w_{g}(t)$ is given by Equation (77), $\tilde{w}_{g}(t):=w_{g}(t) \tilde{H}(t)$ is the particular solution of Equation (75) for the term $f_{\beta}(t) \tilde{H}(t)$, and Lemma 24 states that $\tilde{u}_{f}(t)=D^{\beta} \tilde{w}_{g}(t)$ is the particular solution of Equation (64) for the term $f(t) \tilde{H}(t)$. Lemma 7 shows that $\tilde{u}_{f}(t)=u_{f}(t) \tilde{H}(t)$ if $u_{f}(t)$ is given by $u_{f}(t)={ }_{0} D_{R}^{\beta} w_{g}(t)$, since $\beta \notin \mathbb{Z}_{>-1}$.

\section{Particular Solution of the Hypergeometric Differential Equation}

Let

$$
p_{H}(t, s):=t(1-t) \cdot s^{2}+(c-(a+b+1) t) \cdot s-a b,
$$

where $a \in \mathbb{C}, b \in \mathbb{C}$ and $c \in \mathbb{C}$ are constants. Then the hypergeometric differential equation with an inhomogeneous term $f(t)$ is given by

$$
p_{H}\left(t, \frac{d}{d t}\right) u(t):=t(1-t) \cdot \frac{d^{2}}{d t^{2}} u(t)+(c-(a+b+1) t) \cdot \frac{d}{d t} u(t)-a b \cdot u(t)=f(t), \quad t>0 .
$$

If $f(t)=0$ and $c \notin \mathbb{Z}$, the basic solutions of Equation (87) in $[3,19]$ are given by

$$
\begin{aligned}
& H_{1}(t):={ }_{2} F_{1}(a, b ; c ; t), \\
& H_{2}(t):=t^{1-c} \cdot{ }_{2} F_{1}(1+a-c, 1+b-c ; 2-c ; t),
\end{aligned}
$$

where ${ }_{2} F_{1}(a, b ; c ; z)=\sum_{k=0}^{\infty} \frac{(a)_{k}(b)_{k}}{k !(c) k} z^{k}$ of $z \in \mathbb{C}$ is the hypergeometric series.

We now obtain a particular solution of this equation by the method stated in Section 3 and used in Section 4.1. 
5.1. Green's Function for the Hypergeometric Differential Equation when Condition 2(i) Is Satisfied

The following two lemmas are proved in Section 5.4.

Lemma 28. Let $u(t)$ be the solution of Equation (87), which is expressed by Equation (29). Then the differential equation satisfied by $\tilde{u}(t)=u(t) \tilde{H}(t)$ is given by

$$
p_{H}(t, D) \tilde{u}(t)=f(t) \tilde{H}(t)+u_{0}(c-1) \delta(t) .
$$

Lemma 29. Let $H_{1}(t)$ and $H_{2}(t)$ be given by Equations (88) and (89), and $\psi_{H}(t, \tau)$ for fixed $\tau>0$ be given by

$$
\psi_{H}(t, \tau):=c_{1}(\tau) \cdot H_{1}(t)+c_{2}(\tau) \cdot H_{2}(t),
$$

where $c_{1}(\tau)$ and $c_{2}(\tau)$ are constants which are so chosen that $\psi_{H}(t, \tau)=0$ when $t=\tau$. Then $G_{H}(t, 0)$ and $\mathrm{G}_{H}(t, \tau)$ given by

$$
G_{H}(t, 0)=\frac{1}{c-1} \cdot H_{1}(t) H(t), \quad G_{H}(t, \tau)=\frac{1}{\tau(1-\tau) \psi_{H}^{\prime}(\tau, \tau)} \psi_{H}(t, \tau) H(t-\tau),
$$

are the Green's functions, so that $\tilde{G}_{H}(t, 0)=G_{H}(t, 0) \tilde{H}(t)$ and $\tilde{G}_{H}(t, \tau)=G_{H}(t, \tau) \tilde{H}(t-\tau)$ satisfy

$$
p_{H}(t, D) \tilde{G}_{H}(t, 0)=\delta(t), \quad p_{H}(t, D) \tilde{G}_{H}(t, \tau)=\delta(t-\tau) .
$$

Theorem 4. Let $G_{H}(t, \tau)$ and $\psi_{H}(t, \tau)$ be those given in Lemma 29, Condition 2(i) be satisfied, and $u_{f}(t)$ be given by

$$
u_{f}(t)=\int_{0}^{\infty} G_{H}(t, \tau) f(\tau) d \tau=\int_{0}^{t} \psi_{H}(t, \tau) \frac{f(\tau)}{\tau(1-\tau) \psi_{H}^{\prime}(\tau, \tau)} d \tau
$$

Then $u_{f}(t)$ and $u_{f}(t) \tilde{H}(t)$ are particular solutions of Equations (87) and (90) for the terms $f(t)$ and $f(t) \tilde{H}(t)$, respectively.

Proof. This is confirmed with the aid of Lemma 18.

The condition $\psi_{H}(\tau, \tau)=0$ requires that $c_{1}(\tau) \cdot H_{1}(\tau)=-c_{2}(\tau) \cdot H_{2}(\tau)$, and hence we may choose $\psi_{H}(t, \tau)$ as

$$
\psi_{H}(t, \tau)= \begin{cases}H_{1}(t)-\frac{H_{1}(\tau)}{H_{2}(\tau)} \cdot H_{2}(t), & \left|H_{1}(\tau)\right|<\left|H_{2}(\tau)\right| \\ \frac{H_{2}(\tau)}{H_{1}(\tau)} \cdot H_{1}(t)-H_{2}(t), & \left|H_{1}(\tau)\right| \geq\left|H_{2}(\tau)\right|\end{cases}
$$

5.2. Green's Function for the Hypergeometric Differential Equation in which Condition 2(ii) Is Satisfied

We give the solution of Equation (87) of which the inhomogeneous term $f(t)$ satisfies Condition 2(ii), so that $\tilde{f}(t)=D^{\beta} \tilde{f}_{\beta}(t)$, and $f_{\beta}(t)$ satisfies Condition 2(i).

The following lemma is proved in Section 5.4.

Lemma 30. Let $\tilde{u}(t)$ be a solution of Equation (90), and $p_{\tilde{H}}(t, D)$ be related with $p_{H}(t, D)$ given by Equation (86), by

$$
p_{\tilde{H}}(t, D)=D^{-\beta} p_{H}(t, D) D^{\beta}
$$

Then

$$
p_{\tilde{H}}(t, D):=t(1-t) \cdot D^{2}+(c-\beta-(a+b-2 \beta+1) t) \cdot D-(a-\beta)(b-\beta) .
$$


and $\tilde{w}(t)=D^{-\beta} \tilde{u}(t)$ satisfies

$$
p_{\tilde{H}}(t, D) \tilde{w}(t)=f_{\beta}(t) \tilde{H}(t)+u_{0}(c-1) D^{-\beta} \delta(t) .
$$

Theorem 4 shows that the particular solution of Equation (98) for the term $f_{\beta}(t) \tilde{H}(t)$ is expressed by a particular soltion of

$$
p_{\tilde{H}}\left(t, \frac{d}{d t}\right) w(t)=f_{\beta}(t), \quad t>0 .
$$

That solution is used in giving a solution of Equation (87) in Theorem 5 given below.

Remark 9. We note that $p_{\tilde{H}}(t, D)$ given by Equation (97) is obtained from $p_{H}(t, D)$ given by Equation (86), by replacing $a, b$ and $c$ by $a-\beta, b-\beta$ and $c-\beta$, respectively, and hence the complementary solutions $H_{\beta, 1}(t)$ and $H_{\beta, 2}(t)$ and the Green's functions $G_{\tilde{H}}(t, 0)$ and $G_{\tilde{H}}(t, \tau)$ of Equation (99) are obtained from those $H_{1}(t)$, $H_{2}(t), G_{H}(t, 0)$ and $G_{H}(t, \tau)$, respectively, of Equation (87), by the same replacement.

Now in place of Theorem 4, we have the following theorem, whose proof is given in Section 5.4.

Theorem 5. Let $G_{\tilde{H}}(t, \tau)$ and $\psi_{\tilde{H}}(t, \tau)$ be obtained from $G_{H}(t, \tau)$ and $\psi_{H}(t, \tau)$ by the replacement stated in Remark 9, Condition 2(ii) be satisfied, and $w_{g}(t)$ be given by

$$
w_{g}(t)=\int_{0}^{t} G_{\tilde{H}}(t, \tau) f_{\beta}(\tau) d \tau=\int_{0}^{t} \psi_{\tilde{H}}(t, \tau) \frac{f_{\beta}(\tau)}{\tau \psi_{\tilde{H}}^{\prime}(\tau, \tau)} d \tau
$$

Then $u_{f}(t):={ }_{0} D_{R}^{\beta} w_{g}(t)$ and $u_{f}(t) \tilde{H}(t)$ are particular solutions of Equations (87) and (90) for the terms $f(t)$ and $f(t) \tilde{H}(t)$, respectively.

\subsection{Particular Solution of the Hypergeometric Differential Equation in which Condition 2(iii) Is Satisfied}

We give the solution of Equation (87) of which the inhomogeneous term $f(t)$ satisfies Condition 2(iii), so that $f(t)=g_{v}(t)=\frac{1}{\Gamma(v)} t^{v-1}$ and $\tilde{f}(t)=f(t) \tilde{H}(t)=\hat{f}(D) \delta(t)=\hat{g}_{v}(D) \delta(t)=$ $D^{-v} \delta(t)$. Here we use $\beta \notin \mathbb{Z}_{>-1}$ in place of $-v$.

Lemma 31. Let $\tilde{f}(t)=D^{\beta} \delta(t), p_{\tilde{H}}(t, D)$ be given by Equation (97), and $\tilde{G}_{\tilde{H}}(t, 0)$ satisfy

$$
p_{\tilde{H}}(t, D) \tilde{G}_{\tilde{H}}(t, 0)=\delta(t) .
$$

Then the particular solution of Equation (90) for the term $f(t) \tilde{H}(t)=D^{\beta} \delta(t)$ is given by

$$
\tilde{u}_{f}(t)=D^{\beta} \tilde{G}_{\tilde{H}}(t, 0) .
$$

Proof. $\tilde{u}_{f}(t)$ satisfies $p_{H}(t, D) \tilde{u}_{f}(t)=D^{\beta} \delta(t)$ and hence $p_{\tilde{H}}(t, D) D^{-\beta} \tilde{u}_{f}(t)=\delta(t)$ by Equation (96). Comparing this with Equation (101), we see that Equation (102) is satisfied.

By Remark 9, with the aid of $G_{H}(t, 0)$ given by Equations (92) and (88), we have

Lemma 32. As the solution of Equation (101), we obtain

$$
\tilde{G}_{\tilde{H}}(t, 0)=G_{\tilde{H}}(t, 0) \tilde{H}(t), \quad G_{\tilde{H}}(t, 0)=\frac{1}{c-\beta-1} \cdot{ }_{2} F_{1}(a-\beta, b-\beta ; c-\beta ; t) .
$$


Theorem 6. Let Condition 2(iii) be satisfied, and $G_{\tilde{H}}(t, 0)$ be given by Equation (103). Then the particular solution of Equation (87) is given by

$$
u_{f}(t)={ }_{0} D_{R}^{\beta} G_{\tilde{H}}(t, 0)=\frac{t^{-\beta}}{(c-\beta-1) \Gamma(1-\beta)} \cdot{ }_{3} F_{2}(1, a-\beta, b-\beta ; 1-\beta, c-\beta ; t),
$$

where ${ }_{3} F_{2}\left(a_{1}, a_{2}, a_{3} ; c_{1}, c_{2} ; z\right)=\sum_{k=0}^{\infty} \frac{\left(a_{1}\right)_{k}\left(a_{2}\right)_{k}\left(a_{3}\right)_{k}}{k !\left(c_{1}\right)_{k}\left(c_{2}\right)_{k}} z^{k}$.

Proof. $\tilde{u}_{f}(t)$ is given by Equations (102) and (103). Lemma 7 shows that $\tilde{u}_{f}(t)=u_{f}(t) \tilde{H}(t)$ if $u_{f}(t)$ is given by (104), since $\beta \notin \mathbb{Z}_{>-1}$.

\subsection{Proofs of Lemmas $28-30$ and Theorem 5}

Proof of Lemma 28. Equation (90) is a special one of (48), where $p_{n}, n, a_{2}(t), a_{1}(t)$ and $a_{0}(t)$ stand for $p_{H}, 2, t(1-t), c-(a+b+1) t$ and $-a b$, respectively. Hence by using Equation (37), we see that the second term on the rhs of Equation (48) becomes

$$
\begin{aligned}
\sum_{l=1}^{2} a_{l}(t)\left\langle D^{l} \hat{u}(D)\right\rangle_{0} \delta(t) & =t(1-t)\left(u_{0} D+u_{1}\right) \delta(t)+(c-(a+b+1) t) u_{0} \delta(t) \\
& =u_{0}(D[t(1-t) \delta(t)]-(1-2 t) \delta(t))+c u_{0} \delta(t)=u_{0}(c-1) \delta(t) .
\end{aligned}
$$

We prepare the following lemma.

Lemma 33. Let $\tau \geq 0, p_{H}\left(t, \frac{d}{d t}\right) \psi(t) \cdot H(t-\tau) \in \mathcal{L}_{\text {loc }}^{1}(\mathbb{R})$ and $\tilde{\psi}_{\tau}(t)=\psi(t) \tilde{H}(t-\tau)$. Then

$$
p_{H}(t, D) \tilde{\psi}_{\tau}(t)=p_{H}\left(t, \frac{d}{d t}\right) \psi(t) \cdot \tilde{H}(t-\tau)+\sum_{l=1}^{2} a_{l}(t)\left\langle D^{l} \hat{\psi}_{\tau}(D)\right\rangle_{0} \delta(t-\tau),
$$

where $a_{2}(t)=t(1-t), a_{1}(t)=c-(a+b+1) t, a_{0}(t)=-a b$, and

$$
\begin{aligned}
\sum_{l=1}^{2} a_{l}(t)\left\langle D^{l} \hat{\psi}_{\tau}(D)\right\rangle_{0} \delta(t-\tau)= & \tau(1-\tau) \psi^{\prime}(\tau) \delta(t-\tau)+\psi(\tau)(c-1-(a+b-1) \tau) \delta(t-\tau) \\
& +\psi(\tau) \tau(1-\tau) D \delta(t-\tau) .
\end{aligned}
$$

Proof. Equation (106) is a special one of Equation (18) in Corollary 3. The lhs of Equation (107) is evaluated with the aid of Equation (16) as follows:

$$
\begin{aligned}
& \sum_{l=1}^{2} a_{l}(t)\left\langle D^{l} \hat{\psi}_{\tau}(D)\right\rangle_{0} \delta(t-\tau)=t(1-t)\left[\psi^{\prime}(\tau)+\psi(\tau) D\right] \delta(t-\tau)+(c-(a+b+1) t) \psi(\tau) \delta(t-\tau) \\
& =\left[t(1-t) \psi^{\prime}(\tau)+\psi(\tau)(c-(a+b+1) t)\right] \delta(t-\tau)+\psi(\tau) t(1-t) D \delta(t-\tau) \\
& =\tau(1-\tau) \psi^{\prime}(\tau) \delta(t-\tau)+\psi(\tau)(c-1-(a+b-1) \tau) \delta(t-\tau)+\psi(\tau) \tau(1-\tau) D \delta(t-\tau)
\end{aligned}
$$

where Equation (19) with $h(t)$ and $\tilde{u}(t)$ replaced by $t(1-t)$ and $\delta(t-\tau)$, respectively, is used in the last term of the third member.

Proof of Lemma 29. If we put $\psi(t)=\frac{1}{c-1} \cdot H_{1}(t)$ and $\tau=0$ in Equation (106), then we have $\tilde{\psi}_{\tau}(t)=\tilde{G}(t, 0)$ on the lhs, and the rhs is $\psi(0)(c-1) \delta(t)=\delta(t)$, since $H_{1}(0)=1$. If we put $\psi(t)=\frac{1}{\tau(1-\tau) \psi_{H}^{\prime}(\tau, \tau)} \psi_{H}(t, \tau)$ and $\psi(\tau)=0$ in Equation (106), then we have $\tilde{\psi}_{\tau}(t)=\tilde{G}(t, 0)$ on the lhs, and the rhs is $\tau(1-\tau) \psi^{\prime}(\tau) \delta(t-\tau)=\delta(t-\tau)$. 
Proof of Lemma 30. With the aid of Equations (44) and (45), we obtain

$$
\begin{aligned}
& D^{-\beta}\left[p_{H}(t, D)\left[D^{\beta} \tilde{w}(t)\right]\right]=D^{-\beta}\left[t(1-t) \cdot D^{2}+(c-(a+b+1) t) \cdot D-a b\right]\left[D^{\beta} \tilde{w}(t)\right] \\
& =\left[(t \cdot D-\beta) D-\left(t^{2} \cdot D^{2}-2 \beta t D+\beta(\beta+1)\right)+c \cdot D-(a+b+1)(t \cdot D-\beta)-a b\right] \tilde{w}(t) \\
& =\left[t(1-t) \cdot D^{2}+(c-\beta-(a+b-2 \beta+1) t) \cdot D-(a-\beta)(b-\beta)\right] \tilde{w}(t)=p_{\tilde{H}}(t, D) \tilde{w}(t),
\end{aligned}
$$

which gives Equation (96). When $\tilde{u}(t)=D^{\beta} \tilde{w}(t)$, Equation (90) shows that the lhs of (108) is equal to $D^{-\beta}\left[\tilde{f}(t)+u_{0}(c-1) \delta(t)\right]$ and hence Equation (108) gives Equation (98).

Proof of Theorem 5. Theorem 4 states that when $w_{g}(t)$ is given by Equation $(100), \tilde{w}_{g}(t):=w_{g}(t) \tilde{H}(t)$ is the particular solution of Equation (98) for the term $f_{\beta}(t) \tilde{H}(t)$, and Lemma 30 states that $\tilde{u}_{f}(t)=D^{\beta} \tilde{w}_{g}(t)$ is the particular solution of Equation (90) for the term $f(t) \tilde{H}(t)$. Lemma 7 shows that $\tilde{u}_{f}(t)=u_{f}(t) \tilde{H}(t)$ if $u_{f}(t)$ is given by $u_{f}(t)={ }_{0} D_{R}^{\beta} w_{g}(t)$, since $\beta \notin \mathbb{Z}_{>-1}$.

\section{Solution of Inhomogeneous Differential Equations with Constant Coefficients}

In Section 3 we discuss the solution of an inhomogeneous differential equation with constant coefficients, which takes the form of Equation (56), in terms of the Green's function and distribution theory. In this and next sections, we discuss it in terms of the Green's function and the Laplace transform.

Lemma 34. Let $f(t)$ have the AC-Laplace transform $\hat{f}(s)=\mathcal{L}_{H}[f(t)]$. Then the solution $u(t)$ of Equation (56) has the AC-Laplace transform $\hat{u}(s)=\mathcal{L}_{H}[u(t)]$, which satisfies

$$
p_{n}(s) \hat{u}(s):=\sum_{l=0}^{n} a_{l} s^{l} \hat{u}(s)=\hat{f}(s)+\sum_{l=1}^{n} a_{l}\left\langle s^{l} \hat{u}(s)\right\rangle_{0},
$$

where $\left\langle s^{l} \hat{u}(s)\right\rangle_{0}$ are given by Equations (36) and (37) with D replaced by s.

Proof. This is confirmed with the aid of Lemma 12.

We introduce the Green's function $G(t)$ so that its Laplace transform $\hat{G}(s)$ satisfies

$$
p_{n}(s) \hat{G}(s)=1,
$$

and hence $\hat{G}(s)=\frac{1}{p_{n}(s)}$. Multiplying this to Equation (109), we obtain

$$
\hat{u}(s)=\hat{G}(s) \hat{f}(s)+\hat{G}(s) \sum_{l=1}^{n} a_{l}\left\langle s^{l} \hat{u}(s)\right\rangle_{0} .
$$

Comparing Equations (110) and (109), we see that the differential equation for the Green's function $G(t)$, whose Laplace transform $\hat{G}(s)$ satisfies Equation (110), is Equation (60), and the initial values of $G(t)$ and its derivatives satisfy $\langle p(s) \hat{G}(s)\rangle_{0}=1$, and hence are given by Equation (61). Thus we confirm Lemma 20.

By the inverse Laplace transform of Equation (111), we obtain Lemma 21.

\subsection{Solution of an Inhomogeneous Differential Equation of the First Order}

We consider an inhomogeneous differential equation of the first order:

$$
p_{1}\left(\frac{d}{d t}\right) u(t):=\frac{d}{d t} u(t)+c u(t)=f(t), \quad t>0
$$

where $c \in \mathbb{C}$ is a constant. 
By Lemma 34, we obtain the following equation for $\hat{u}(s)=\mathcal{L}_{H}[u(t)]$ :

$$
p_{1}(s) \hat{u}(s)=(s+c) \hat{u}(s)=u_{0}+\hat{f}(s) .
$$

Following Section 6, we introduce the Green's function $G(t)$, so that its Laplace transform $\hat{G}(s)$, which satisfies Equation (110), is given by $p_{1}(s) \hat{G}(s)=(s+c) \cdot \hat{G}(s)=1$, and hence we have

$$
\hat{G}(s)=\frac{1}{p_{1}(s)}=\frac{1}{s+c}
$$

By using this equation in Equation (113) and putting $\hat{u}_{f}(s)=\hat{G}(s) \hat{f}(s)$, we obtain

$$
\hat{u}(s)=u_{0} \hat{G}(s)+\hat{u}_{f}(s), \quad \hat{u}_{f}(s)=\hat{G}(s) \hat{f}(s) .
$$

By the inverse Laplace transform of Equation (114), we obtain

$$
G(t)=e^{-c t} H(t)
$$

Theorem 7. Let Condition 2(i) or 2(ii) be satisfied. Then the solution of Equation (112) is given by

$$
u(t)=u_{0} G(t)+u_{f}(t), \quad t>0,
$$

where

$$
u_{f}(t)=\int_{0}^{t} G(t-\tau) f(\tau) d \tau=e^{-c t} \int_{0}^{t} e^{c \tau} f(\tau) d \tau, \quad t>0
$$

or

$$
u_{f}(t)={ }_{0} D_{R}^{\beta}\left[\int_{0}^{t} G(t-\tau) f_{\beta}(\tau) d \tau\right]={ }_{0} D_{R}^{\beta}\left[e^{-c t} \int_{0}^{t} e^{c \tau} f_{\beta}(\tau) d \tau\right], \quad t>0,
$$

according as Condition 2(i) or 2(ii) is satisfied.

Proof. By the AC-Laplace transform of Equation (117), we obtain Equation (115). When Condition 2(i) is satisfied, the Laplace transform of Equation (118) is $\hat{u}_{f}(s)=\hat{G}(s) \hat{f}(s)$. When Condition 2(ii) is satisfied, we confirm that the AC-Laplace transform of Equation (119) is $\hat{u}_{f}(s)=s^{\beta} \hat{G}(s) \hat{f}_{\beta}(s)$, with the aid of Equations (28) and (27).

Theorem 8. Let Condition 2(iii) be satisfied, so that $\hat{f}(s)=s^{\beta}$ for $\beta \in \mathbb{C} \backslash \mathbb{Z}>-1$. Then the solution of Equation (112) is given by Equation (117) with

$$
u_{f}(t)=t^{-\beta} \sum_{k=0}^{\infty} \frac{(1)_{k}(-c t)^{k}}{k ! \Gamma(k+1-\beta)}=\frac{t^{-\beta}}{\Gamma(1-\beta)} \cdot{ }_{1} F_{1}(1 ; 1-\beta ;-c t)
$$

Proof. By using Equation (114) in Equation (115), we have

$$
\hat{u}_{f}(s)=\frac{s^{\beta}}{s+c}=\sum_{k=0}^{\infty}(-c)^{k} s^{-k-1+\beta}
$$

By the inverse Laplace transform of this equation, we obtain Equation (120), where we use $\Gamma(k+1-\beta)=\Gamma(1-\beta)(1-\beta)_{k}$. 
6.2. Solution of an Inhomogeneous Differential Equation of the Second Order

We consider an inhomogeneous differential equation of the second order:

$$
p_{2}\left(\frac{d}{d t}\right) u(t):=\frac{d^{2}}{d t^{2}} u(t)+b \frac{d}{d t} u(t)+c u(t)=f(t), \quad t>0
$$

where $b \in \mathbb{C}$ and $c \in \mathbb{C}$ are constants.

By Lemma 34, we obtain the following equation for $\hat{u}(s)=\mathcal{L}_{H}[u(t)]$ :

$$
p_{2}(s) \hat{u}(s):=\left(s^{2}+b s+c\right) \hat{u}(s)=\left(u_{0} s+u_{1}\right)+b u_{0}+\hat{f}(s) .
$$

Following Section 6, we introduce the Green's function $G(t)$, so that its Laplace transform $\hat{G}(s)$, which satisfies Equation (110), is given by $p_{2}(s) \hat{G}(s)=\left(s^{2}+b s+c\right) \cdot \hat{G}(s)=1$, and hence we have

$$
\hat{G}(s)=\frac{1}{p_{2}(s)}=\frac{1}{s^{2}+b s+c} .
$$

By using Equation (124) in Equation (123), we obtain

$$
\hat{u}(s)=u_{0} s \hat{G}(s)+\left(u_{1}+b u_{0}\right) \hat{G}(s)+\hat{u}_{f}(s),
$$

where

$$
\hat{u}_{f}(s)=\hat{G}(s) \hat{f}(s) \text {. }
$$

Equation:

$$
p_{2}(s):=s^{2}+b s+c=0
$$

has one or two roots according as $c=\frac{b^{2}}{4}$ or not. If $c \neq \frac{b^{2}}{4}$, then Equation (127) has two different roots $\mu_{1}$ and $\mu_{2}$, so that $b=-\left(\mu_{1}+\mu_{2}\right)$ and $c=\mu_{1} \mu_{2}$, and Equation (124) gives

$$
\hat{G}(s)=\frac{1}{\left(s-\mu_{1}\right)\left(s-\mu_{2}\right)}=\frac{1}{\mu_{1}-\mu_{2}}\left(\frac{1}{s-\mu_{1}}-\frac{1}{s-\mu_{2}}\right) .
$$

By the inverse Laplace transform, we then obtain

$$
G(t)=\frac{1}{\mu_{1}-\mu_{2}}\left(e^{\mu_{1} t}-e^{\mu_{2} t}\right) H(t)
$$

If $c=\frac{b^{2}}{4}$, then Equation (127) has only one root $\mu_{1}$, so that $b=-2 \mu_{1}$ and $c=\mu_{1}^{2}$, and in place of Equation (128), we have

$$
\hat{G}(s)=\frac{1}{\left(s-\mu_{1}\right)^{2}} .
$$

By the inverse Laplace transform, we then obtain

$$
G(t)=t e^{\mu_{1} t} H(t)
$$

Theorem 9. Let Condition 2(i) or 2(ii) be satisfied. Then the solution of Equation (122) is given by

$$
u(t)=u_{0} \frac{d}{d t} G(t)+\left(u_{1}+b u_{0}\right) G(t)+u_{f}(t), \quad t>0
$$


where

$$
u_{f}(t)=\int_{0}^{t} G(t-\tau) f(\tau) d \tau, \quad t>0
$$

or

$$
u_{f}(t)={ }_{0} D_{R}^{\beta}\left[\int_{0}^{t} G(t-\tau) f_{\beta}(\tau) d \tau\right], \quad t>0,
$$

according as Condition 2(i) or 2(ii) is satisfied. If $c \neq \frac{b^{2}}{4}$, then $b=-\left(\mu_{1}+\mu_{2}\right), c=\mu_{1} \mu_{2}$, and $G(t)$ given by Equation (129) is used in Equation (132). If $c=\frac{b^{2}}{4}$, then $b=-2 \mu_{1}, c=\mu_{1}^{2}$, and $G(t)$ given by Equation (131) is used there.

Proof. By the AC-Laplace transform of Equation (132), we obtain Equation (125). When Condition 2(ii) is satisfied, we have $\hat{f}(s)=s^{\beta} \hat{f}_{\beta}(s)$. By using Equations (28) and (27), we confirm that the AC-Laplace transform of $u_{f}(t)$ given by Equation (134) is $s^{\beta} \hat{G}(s) \hat{f}_{\beta}(s)$.

Theorem 10. Let Condition 2(iii) be satisfied, be satisfied, so that $\hat{f}(s)=s^{\beta}$ for $\beta \in \mathbb{C} \backslash \mathbb{Z}_{>-1}$. Then the solution of Equation (122) is given by Equation (132) with $u_{f}(t)$ given as follows. If $c \neq \frac{b^{2}}{4}$, then $b=-\left(\mu_{1}+\mu_{2}\right)$, $c=\mu_{1} \mu_{2}$, and

$$
u_{f}(t)=\frac{1}{\mu_{1}-\mu_{2}} \frac{t^{-\beta}}{\Gamma(1-\beta)}\left[{ }_{1} F_{1}\left(1 ; 1-\beta ; \mu_{1} t\right)-{ }_{1} F_{1}\left(1 ; 1-\beta ; \mu_{2} t\right)\right] .
$$

If $c=\frac{b^{2}}{4}$, then $b=-2 \mu_{1}, c=\mu_{1}^{2}$, and

$$
u_{f}(t)=\frac{t^{1-\beta}}{\Gamma(1-\beta)} \cdot{ }_{1} F_{1}\left(2 ; 1-\beta ; \mu_{1} t\right)
$$

Proof. When $c \neq \frac{b^{2}}{4}$, by using Equation (128) in Equation (126), we have

$$
\hat{u}_{f}(s)=\frac{1}{\mu_{1}-\mu_{2}}\left(\frac{s^{\beta}}{s-\mu_{1}}-\frac{s^{\beta}}{s-\mu_{2}}\right) .
$$

In the proof of Theorem 8, we obtain Equation (120), by the inverse Laplace transform of $\hat{u}_{f}(s)$ given by Equation (121). By the corresponding inverse Laplace transform of Equation (137), we obtain Equation (135). When $c=\frac{b^{2}}{4}$, by using Equation (130), we have

$$
\hat{u}_{f}(s)=-s^{\beta} \frac{d}{d s} \frac{1}{s-\mu_{1}}=-s^{\beta} \frac{d}{d s} \sum_{k=0}^{\infty} \mu_{1}^{k} s^{-k-1}=s^{\beta} \sum_{k=0}^{\infty} \mu_{1}^{k}(k+1) s^{-k-2} .
$$

By the inverse Laplace transform, we have

$$
u_{f}(t)=t^{1-\beta} \sum_{k=0}^{\infty} \frac{(k+1)\left(\mu_{1} t\right)^{k}}{\Gamma(k+1-\beta)}=\frac{t^{1-\beta}}{\Gamma(1-\beta)} \sum_{k=0}^{\infty} \frac{(2)_{k}\left(\mu_{1} t\right)^{k}}{k !(1-\beta)_{k}},
$$

which gives Equation (136).

\subsection{Application of the Theorems in Section 6.1}

We consider an inhomogeneous differential equation with polynomial coefficients of the first order:

$$
\frac{d}{d x} w(x)-2 x w(x)=2 x g(x)=1, \quad x \in \mathbb{R}
$$


We put $t=x^{2}, u(t)=u\left(x^{2}\right)=w(x)$ and $f(t)=f\left(x^{2}\right)=g(x)$. Then $u(t)$ satisfies

$$
\frac{d}{d t} u(t)-u(t)=f(t), \quad t \in \mathbb{R}_{>0}
$$

where

$$
f(t)=\frac{1}{2 x}=\frac{t^{-1 / 2}}{2}=\frac{\Gamma(1 / 2)}{2} g_{1 / 2}(t)=\frac{\sqrt{\pi}}{2} g_{1 / 2}(t)
$$

where $g_{1 / 2}(t)$ is defined by Equation (26).

Lemma 35. Let $u(t)$ be a solution of Equation (141). Then $w(x)=u\left(x^{2}\right)$ gives a solution of Equation (140).

Lemma 36. The solution of Equation (141) is given by Equation (117), where $u_{0}=u(0)$ and

$$
G(t)=e^{t}, \quad u_{f}(t)=\frac{\sqrt{\pi}}{2} \sum_{k=0}^{\infty} \frac{t^{k+1 / 2}}{\Gamma\left(k+\frac{3}{2}\right)}, \quad t \in \mathbb{R}_{>0} .
$$

The solution of Equation (140) is given by

$$
w(x)=w_{0} G_{w}(x)+w_{g}(x), \quad x \in \mathbb{R}
$$

where $w_{0}=w(0)$ and

$$
G_{w}(x)=e^{x^{2}}, \quad w_{g}(x)=\frac{\sqrt{\pi}}{2} \sum_{k=0}^{\infty} \frac{x^{2 k+1}}{\Gamma\left(k+\frac{3}{2}\right)}, \quad x \in \mathbb{R}
$$

Proof. By using $c=-1, \beta=\frac{1}{2}$, Equation (142) in Equations (116) and (120), we see that the solution of Equation (141) is given by Equation (117) with Equation (143). Now the solution of Equation (140) is obtained from it with the aid of Lemma 35.

Lemma 37. The asymptotic behavior of $u_{f}(t)$ and $w_{g}(x)$ are given by

$$
\begin{gathered}
u_{f}(t)=\frac{\sqrt{\pi}}{2} e^{t}-\sum_{k=0}^{\infty} \frac{(-1)^{k}\left(-\frac{1}{2}\right)_{k}}{2 t^{k+1 / 2}}, \quad t \rightarrow \infty, \\
w_{g}(x)= \begin{cases}\frac{\sqrt{\pi}}{2} e^{x^{2}}-\sum_{k=0}^{\infty} \frac{(-1)^{k}\left(-\frac{1}{2}\right)_{k}}{2 x^{2 k+1}}, & x \rightarrow \infty, \\
-\frac{\sqrt{\pi}}{2} e^{x^{2}}-\sum_{k=0}^{\infty} \frac{(-1)^{k}\left(-\frac{1}{2}\right)_{k}}{2 x^{2 k+1}}, & x \rightarrow-\infty .\end{cases}
\end{gathered}
$$

Proof. By using Equations (142) and (143) in Equation (118), we have

$$
u_{f}(t)=\int_{0}^{t} e^{t-\tau} \frac{\tau^{-1 / 2}}{2} d \tau=e^{t} \frac{\Gamma\left(\frac{1}{2}\right)}{2}-\frac{1}{2} \int_{0}^{\infty} e^{-\tau}(t+\tau)^{1 / 2} d \tau=e^{t} \frac{\sqrt{\pi}}{2}-\frac{1}{2} \sum_{k=0}^{\infty} \frac{(-1)^{k}\left(-\frac{1}{2}\right)_{k}}{t^{k+1 / 2}}
$$

which gives Equation (146). Equation (147) is obtained from it with the aid of Lemma 35.

Equation (145) shows that the particular solution $w_{g}(x)$ of Equation (140) is an odd function of $x$. As a consequence of this fact, the asymptotic behavior of $w_{g}(x)$ is given by Equation (147).

Acknowledgments: The authors are grateful to the reviewers of this paper. Following their suggestions, the last paragraph of Introduction and proofs of some theorems are included, and proofs of some lemmas are rewritten in details.

Author Contributions: In the last paragraph of Introduction, recent study on the solution of differential equations is reviewed. After the paper [2] was published, Tohru Morita wrote a manuscript giving particular solutions of 
Kummer's and the hypergeometric differentail equations in terms of distribution theory and the Green's function. Tohru Morita and Ken-ichi Sato worked together to improve the manuscript to the present paper, where the solution of differential equations with constant coefficients is written in the form where the role of the Green's function is seen in the framework of the AC-Laplace transform.

Conflicts of Interest: The authors declare no conflict of interest.

\section{References}

1. Morita, T.; Sato, K. Solution of Differential Equations with the Aid of Analytic Continuation of Laplace Transform. Appl. Math. 2014, 5, 1309-1319.

2. Morita, T.; Sato, K. Solution of Differential Equations of Polynomial Coefficients with the Aid of Analytic Continuation of Laplace Transform. Mathematics 2016, 4, 19.

3. Abramowitz, M.; Stegun, I.A. Handbook of Mathematical Functions with Formulas, Graphs and Mathematical Tables; Dover Publ., Inc.: New York, NY, USA, 1972; Chapter 13.

4. Morita, T.; Sato, K. Asymptotic Expansions of Fractional Derivatives and Their Applications. Mathematics 2015, 3, 171-189.

5. Zemanian, A.H. Distribution Theory and Transform Analysis; Dover Publ., Inc.: New York, NY, USA, 1965.

6. Godoy, E.; Ronveaux, A.; Zarzo, A.; Area, I. Connection Problems for Polynomial Solutions of Nonhomogeneous Differental and Difference Equations. J. Comput. Appl. Math. 1998, 99, 177-187.

7. Morita, T.; Sato, K. Remarks on the Solution of Laplace's Differential Equation and Fractional Differential Equation of That Type. Appl. Math. 2013, 4, 13-21.

8. Morita, T.; Sato, K. Solution of Laplace's Differential Equation and Fractional Differential Equation of That Type. Appl. Math. 2013, 4, 26-36.

9. Yosida, K. Operational Calculus; Springer: New York, NY, USA, 1982; Chapter VII.

10. Yosida, K. The Algebraic Derivative and Laplace's Differential Equation. Proc. Jpn. Acad. Ser. A 1983, 59, 1-4.

11. Morita, T.; Sato, K. Solution of Fractional Differential Equation in Terms of Distribution Theory. Interdiscip. Inf. Sci. 2006, 12, 71-83.

12. Morita, T.; Sato, K. Neumann-Series Solution of Fractional Differential Equation. Interdiscip. Inf. Sci. 2010, 16, 127-137.

13. Gelfand, I.M.; Shilov, G.E. Generalized Functions; Academic Press Inc.: New York, NY, USA, 1964; Volume 1.

14. Vladimirov, V.S. Methods of the Theory of Generalized Functions; Taylor \& Francis Inc.: New York, NY, USA, 2002.

15. Schwartz, L. Théorie des Distributions; Hermann: Paris, France, 1966.

16. Morita, T.; Sato, K. Liouville and Riemann-Liouville Fractional Derivatives via Contour Integrals. Fract. Calc. Appl. Anal. 2013, 16, 630-653.

17. Podlubny, I. Fractional Differential Equations; Academic Press: San Diego, CA, USA, 1999.

18. Miller, K.S.; Ross, B. An Introduction to the Fractional Calculus and Fractional Differential Equations; John Wiley: New York, NY, USA, 1993.

19. Magnus, M.; Oberhettinger, F. Formulas and Theorems for the Functions of Mathematical Physics; Chelsea Publ. Co.: New York, NY, USA, 1949; Chapter VI. 